\title{
Are Current Theories of Panic Falsifiable?
}

\author{
Walton T. Roth, Frank H. Wilhelm, and Dean Pettit \\ Department of Veterans Affairs Health Care System, Palo Alto, California, and Stanford University School of Medicine
}

\begin{abstract}
The authors examine 6 theories of panic attacks as to whether empirical approaches are capable of falsifying them and their heuristic value. The authors conclude that the catastrophic cognitions theory is least falsifiable because of the elusive nature of thoughts but that it has greatly stimulated research and therapy. The vicious circle theory is falsifiable only if the frightening internal sensations are specified. The 3-alarms theory postulates an indeterminate classification of attacks. Hyperventilation theory has been falsified. The suffocation false alarm theory lacks biological parameters that unambiguously index dyspnea or its distinction between anticipatory and panic anxiety. Some correspondences postulated between clinical phenomena and brain areas by the neuroanatomical hypothesis may be falsifiable if panic does not depend on specific thoughts. All these theories have heuristic value, and their unfalsifiable aspects are capable of modification.
\end{abstract}

Keywords: review, panic, theory, falsifiability, cognition, respiration, neuroanatomy

Theories play an important role in mental health research and practice. In the face of incomplete knowledge, scientifically oriented clinicians and researchers turn to theories for guidance. The rational clinician must choose between treatments whose biochemical or psychological foundations are controversial, and researchers must plan new investigations, the outcomes of which will better explain past observations and better predict future results. However, not all theories are formulated adequately. The philosopher of science Karl Popper criticized the claims of Marxism and psychoanalysis that their theories were unscientific, because they were unfalsifiable. He contended that for theories to be scientific, they had to be formulated so that disproof by negative evidence was possible (Popper, 1959). This thesis is independent of Popper's more controversial claim that empirical evidence can never provide positive support for a theory. Evidence can never confirm a theory, according to Popper; it can only ever disconfirm. This part of Popper's philosophical program is widely contested in the field of philosophy of science. Yet Popper's enduring insight was that unfalsifiability is a vice in scientific theories. A theory cannot be supported by empirical observation, if it is formulated so as to be consistent with any observations whatever. A satisfactory theory is one from which statements or propositions can be derived that potentially can be empirically disconfirmed. These propositions are predictions of, or hypotheses about, how specific empirical observations will turn out. Thus, the preferred argument of the contemporary mental health grant proposal is that a hypothesis will

Walton T. Roth, Frank H. Wilhelm, and Dean Pettit, Department of Veterans Affairs Health Care System, Palo Alto, California, and Department of Psychiatry and Behavioral Sciences, Stanford University School of Medicine.

Preparation of this article was supported by the Department of Veterans Affairs and National Institutes of Health Grant MH56094. We especially thank Georg Alpers for his helpful suggestions.

Correspondence concerning this article should be addressed to Walton T. Roth, VA Palo Alto Health Care System (116F-PAD), 3801 Miranda Avenue, Palo Alto, CA 94304. E-mail: wtroth@stanford.edu be tested. A grant proposal must convince the reviewer committee that the experiments planned and their statistical analysis will allow decisions to be made whether the hypotheses advanced are true. Unless the null hypothesis is disconfirmed at the .05 probability level, investigators are under pressure to modify their original theories or give them up.

Although essential, falsifiability is not the only characteristic of good theory. A better theory is more comprehensive, explaining a broader range of phenomena in more circumstances than alternative theories (Kuhn, 1977), and makes predictions that are contrary to current scientific or lay beliefs. A better theory has heuristic (from the Greek word "to discover") value, meaning that it serves to guide, discover, or reveal, even though it is unproved or incapable of proof. A more heuristic mental health theory stimulates researchers and clinicians to question what they have believed about etiologies and treatments and to be innovative and explore new approaches. We have chosen theories to examine that seem to us to have heuristic value. For some of them, we have examined how many times they have been cited in the scientific literature as objective evidence of their heuristic value, although this criterion has a number of serious limitations. Falsifiability and heuristic value are not completely independent. An unfalsifiable theory should evolve toward greater falsifiability if it is to keep its heuristic value.

Here we attempt to evaluate six theories of panic in the light of Popper's falsifiability criterion and their heuristic value. An initial difficulty is how to define panic, which in ordinary language usually refers to sudden, intense anxiety. Clinically, panic has come to be restricted to anxiety without an obvious immediate triggering stimulus, an anxiety which over time can lead to avoidance of situations that are difficult to leave quickly, and whose somatic symptoms lead to concerns about heart disease or other physical illness. According to the current Diagnostic and Statistical Manual of Mental Disorders (4th ed.; DSM-IV; American Psychiatric Association, 1994), which is based on a medical model with its own theoretical assumptions, panic attacks (PAs) are both a syndrome and an essential component of one anxiety disorder, 
and they apparently occur in other anxiety disorders, in psychiatric disorders that are not anxiety disorders, and in individuals who do not qualify for a diagnosis of a psychiatric disorder at all. In any case, no definition of panic is theory free, and the theories we examine here differ in how distinct from other kinds of anxiety they regard panic to be. Most consider anticipatory anxiety and panic to be basically different, and one (the suffocation false alarm theory [SFAT]) distinguishes fear and anxiety. For all of the theories except perhaps the SFAT, the same explanation for PAs could apply whether or not the attacks occurred in patients with panic disorder (PD).

The theories that we consider-catastrophic cognitions theory (CCT), vicious circle theory (VCT), three-alarms theory (TAT), hyperventilation theory (HVT), SFAT, and Gorman's neuroanatomical hypothesis $(\mathrm{NH})$-all claim explanatory power for the lion's share of attacks of the kind typically experienced by patients with PD. Other things being equal, the more attacks that a theory explains, the more useful it is, but if it only purports to explain a subset, some independent way of classifying panics or panickers as being members of the relevant subset is required. Otherwise, the theory cannot be falsified because any negative results can be attributed to their coming from the wrong subset. In many respects, however, these theories are profoundly different, probably because astoundingly diverse treatments appear to be helpful for panic. These include an array of medications with dissimilar chemical actions (from selective serotonin reuptake inhibitors to benzodiazepines) and a contradictory assortment of psychological approaches, from cognitive-behavioral therapy ([CBT]; Barlow, 1997) to mindfulness meditation (Miller, Fletcher, \& Kabat-Zinn, 1995). CBT packages typically address the client's anxiety with ideas from three theories-catastrophic cognitions, the vicious circle model, and hyperventilation (HV).

Five of the six theories are causal while the sixth is more concerned with brain localization. There could be a variety of causal factors that we might, in certain contexts, be prepared to call a cause or contributing cause of PAs, for example, stressful situations, excessive caffeine intake. However, five of the six theories of PAs considered here aim at identifying the underlying proximal cause of a PA through which all such distal or contributing causes have their effect, if any. Each causal theory of PAs considered here presupposes that there is a unique underlying proximal cause, a single underlying event that is both causally necessary and under normal background conditions causally sufficient for a PA. ${ }^{1}$ In principle, testing such a causal hypothesis is a straightforward matter of determining whether the candidate cause is correlated with PAs. A causal factor that is absent when a PA occurs can be ruled out as the underlying proximal cause of PAs. In practice, however, evidence that a PA has occurred in the absence of the candidate causal factor can be explained away by invoking some auxiliary hypothesis (e.g., the candidate causal factor was present but went undetected by the experimental methodology, or some normal background condition failed to obtain). As Quine (1951) insisted, contra Popper, scientific hypotheses are not tested in isolation. Rather, as Quine put it, scientific hypotheses confront empirical evidence as a "corporate body." Yet each of the causal theories considered in this article purports to give the core mechanism of PAs, the proximal cause through which all other distal and contributing causes have their effect on PAs. Such a cause would be, under normal background conditions, causally sufficient for a PA. If a PA can occur in the absence of the putative cause and the theory attempts to explain this away by postulating modulating or inhibiting factors, then the theory is weakened insofar as it would fail to identify the core mechanism of PAs.

Moreover Popper's worry still stands: A theory should not be formulated in such a way that no evidence could ever count as disconfirmation of it. Immunity to falsification is a vice of a theory, not a virtue. If we could not in principle have evidence that a PA occurred in the absence of the candidate causal factor, then the theory cannot be subjected to any meaningful empirical test.

Whether the cause of panic is hypothesized to be catastrophic thoughts, feared somatic sensations, a low arterial $\mathrm{pCO}_{2}$ ("p" refers to partial pressure), or a suffocation false alarm, the testing of such causal theories follows similar logic. For a hypothesis about the cause of PAs to be falsifiable requires at least that the criteria for the occurrence of the putative cause be independent of the occurrence of the PAs. If there are no criteria for the occurrence of the putative cause other than the occurrence of PA, then there could not even in principle be evidence that a PA occurred in the absence of the putative cause. Hence, in what follows, we generally evaluate these theories with questions like these: Can the occurrence of putative cause be precisely and objectively established independent of a PA? If the putative cause occurs naturally, will a PA follow it, and if a PA occurs naturally, will the putative cause have immediately proceeded or accompanied it? If the putative cause is induced, will a PA follow it, and if a PA is induced, will the putative cause have preceded or accompanied it? If the putative cause is suppressed, will a PA be suppressed, and if a PA is suppressed, will the putative cause preceding it have been suppressed.

Previous reviews taking this approach concentrated on CCT (Hofmann, 2003; McNally, 1994, 2001), and here we extend it to rival theories. Theories that do not admit of disconfirming evidence of this sort are unfalsifiable, and hence cannot be subjected to meaningful empirical test. Although Popper thought that falsifiability should apply only to the formulation of the theory and not to whether empirical methods that could falsify it are available or are likely to become available, we try to address both issues. In our opinion, some currently pursued approaches for testing are logically flawed while others would require improvements in methodology that may be long in coming. In any case, we doubt if any of these theories of PAs will be overthrown completely by a single decisive experiment. The way science usually works is that negative results first lead to modifications of the original theory but at the same time make more attractive alternative theories that are able to explain a few more of the available data (Putnam, 1974). In

\footnotetext{
${ }^{1}$ A cause is never sufficient by itself for its effect. The striking of the match that causes the fire is not sufficient for the fire, because the presence of oxygen is also required. At best, a cause is what Mackie (1965) called an "INUS condition"-an individually necessary part of a set of conditions that is sufficient for the effect but not necessary for it (since the effect might have been produced by a different set of conditions). In this case, since the theories of concern here purport to give the unique proximal cause of PA, the candidate cause should be causally necessary for PAs. All claims here about necessity and sufficiency should obviously be understood as causal necessity and causal sufficiency. The claim that a putative cause is sufficient for its effect should be understood as the claim that it sufficient under normal background conditions.
} 
addition, because empirical observations are imperfect, evidence from any data set at best affects only the probability that a theory is true; explanations will never be consistent with every observation that has been reported. Often the "weight of the evidence" is referred to in concluding arguments.

Note that we use variations of the word prove in many places in this article. Strictly speaking, proofs are only possible for abstract formulations: In the empirical world, proof is relative, a matter of probability. In statistics, probability levels can be specified, but for the more general assertions that we discuss here, that is not feasible. We use proved in the vague way that it is used in ordinary language, to mean that many but not necessarily all experts on the matter would be convinced.

\section{CCT}

\section{Core Thesis}

This theory was proposed by Clark (1986) in an article that became the second-most-cited article in psychology among the more than 50,000 published between 1986 and 1990 (Garfield, 1992). Clark's core thesis is that PAs are caused by catastrophic beliefs about certain internal bodily sensations or external stimuli, which make the panicker react to these stimuli in certain contexts with immediate, extreme anxiety. A typical example of a catastrophic cognition is on noticing sensations from the pumping of the heart, the panicker will have the terrifying thought "I'm having a heart attack and might die at any moment." Internal stimuli that "arise from perception of a mental process," for example, one's mind going blank, are also possible triggers. The triggers most often cited as examples are body sensations that accompany anxiety. These triggers are thus part of a causal chain of somatic sensations, which cause catastrophic thoughts, which in turn cause PAs. There are other possible causes of catastrophic thoughts, but catastrophic thoughts are necessary for PAs to occur.

Clark's (1986) formulation includes a positive feedback loop or vicious circle between bodily sensations accompanying anxiety and anxiety generated from perceiving them. We discuss this separately under the rubric of the VCT, since it is not specific to the idea of catastrophic cognitions (it has been used to supplement HVT), and has older historical antecedents. Not all stimuli that trigger catastrophic cognitions are potential elements of a vicious circle, for example, internal stimuli that are not part of the somatic anxiety response and external stimuli such as agoraphobic situations where attacks had occurred previously.

Clark in his 1986 article acknowledged the possibility of biological vulnerability factors but considered catastrophic thoughts necessary for an attack. For example, a physiological trait characterized by more intense or more abruptly fluctuating bodily states, although medically benign, could conduce to panic in people with catastrophic attitudes about their bodily functions. One mechanism of pharmacotherapy could be to block such fluctuations. In a later article with Ehlers, he mentions that another kind of attack without catastrophic misinterpretations could occur, usually early in the course of the illness (Clark \& Ehlers, 1993). Here we critique only the "strong" version of the theory, where catastrophic misinterpretations are necessary, since without an independent criterion of what kind of attack is occurring, falsification would be stymied.
Tests

Whether CCT measures up to Popper's and others' falsifiability standards has been recently discussed by Hofmann (2003), who concludes that at best this theory is difficult to test. McNally has reviewed the explanatory limitations of CCT in particular (McNally, 1994) and of cognitive appraisal models of anxiety disorder in general (McNally, 2001). Clark anticipated some of these and other criticisms in his 1986 article, not only by presenting the evidence that he considered confirmatory but also by providing doubters three specific predictions to test CCT's central premises-that panickers will interpret bodily sensations in a catastrophic way, that provocations will only produce panic when the sensations are interpreted catastrophically, and that treatments that fail to change catastrophic thinking will have higher relapse rates. Here is our version of essential propositions that are important for CCT and our opinion about how well they fare under critical scrutiny. Note that in our discussion we refer to cognitive contents as thoughts or cognitions, which is different from cognitive processes.

1. The occurrence of catastrophic thoughts can be precisely and objectively established independent of a PA.

If we cannot be sure whether catastrophic thoughts are really absent or present independent of the subject's knowledge of CCT and are not able to separate these thoughts from the PA itself, CCT is unfalsifiable as a causal explanation of panic. Some critics reject categorically any theory based on thoughts as unworthy of scientific consideration, because there is too much uncertainty about the content of our own and other people's minds. If the behaviorist movement in psychology and biological treatment in psychiatry have meant progress, theoretical explanations like those of Clark's are atavistic. One cannot be sure that thoughts reported to be absent really were absent, since they may not have been noticed, been noticed and forgotten, or were unconscious or in some other way incomplete, unformed, or inaccessible. An inquiry about thoughts may be misunderstood or may bias the answer. Even methods for assessing thoughts and attitudes that do not ask for them directly, such as reaction time tasks or brain potentials, may not overcome the limitations of self-report. There is little evidence that such methods penetrate through conscious or unconscious deception to reach hidden "true" thoughts. Therapy situations are especially likely to produce biased information. The rationale of CBT is explicitly communicated to PD patients in its initial sessions. The expectation is generated that catastrophic thoughts must be present if panic occurs. Examples of such thoughts are given along with the message that clients must identify their own catastrophic thoughts and learn to counteract them if relief from attacks is to be obtained. Thus, affirmations of having catastrophic thoughts during panic after such thoughts had been initially denied is particularly weak evidence for CCT. On the other hand, denials cannot be accepted at face value either.

According to Lang's (1988) bioinformational theory of emotion, cognitions are an integral part of a neural network that includes physiological and behavioral elements. For the panicker, the thought "I am about to die" and a racing heart form a single, contemporaneous entity. Although this does not rule out changing the physiological and behavioral elements of an emotion by chang- 
ing its semantic components, it does mean that these elements may not be separable temporally, without which we cannot infer the direction of causality from observations of the sequence of emotional events. We could never establish that certain thoughts cause the physiological manifestations of panic rather than vice versa by observing that these thoughts regularly precede these manifestations because they would always be coexisting, simultaneously present aspects of a single network. Yet, our conceptions of neural networks are tentative. Phenomenologically, thoughts and feelings are sometimes experienced as occurring serially, so a casual theory might be justified based on the serial activation of different networks or different parts of the same network. Such a theory could be conceptualized as having no one necessary and sufficient cause but multiple contributing causes of which none is necessary or sufficient but that combine to result in a PA.

Parenthetically, the fact that the thoughts accompanying PAs may be different from those accompanying other kinds of anxiety is not a convincing argument for cognition and other aspects of emotion being separable. PAs are defined as having an acute onset in the absence of an obvious phobic stimulus, and this definition limits the kinds of thoughts that will logically associate with these attacks. Acute anxiety implies an acute threat, and the absence of external sources of danger implies internal sources, the exact nature of which is uncertain. Cognitions associated with panic are often speculations about the source of the threat and ways of being safe from it. That the cognitions fit the anxiety should be no surprise and has no causal implications.

The difficulty of separating cognition from other aspects of emotion impairs not only our ability to temporally sequence these aspects of emotion but also our capacity to judge the potency of a specific thought for causing a PA. Surely not every momentary health worry will precipitate a PA. Causes must have magnitudes related to the size of their presumed effect. Suppose, for example, that a panicker reports the thought "My heart is pounding, which could be a sign of heart disease and which means I might pass out in the next minute, although I have had these attacks before and nothing has happened so far." How accurately can we rate the affective impact of this thought as a cold cognition without the circularity of having to know how frightened the individual is when having the thought? Its potency in causing a PA must depend on how strongly the individual believes that heart pounding is a sign of heart disease, how likely syncope will occur, and how threatening syncope is. But ordinarily the strength of these factors would be judged by establishing how much fear was present at the time of the thought, begging the question of the causal relationship between thought and emotion. In fact, the relevant thought cannot be observed as a pure semantic entity. When calm, a panic patient might report thinking, "Inexplicable heart pounding could be sign of heart disease," while in a moment of panic, dire outcomes seem both more plausible and more probable, and the thought will be different, for example, "My heart's pounding which means I may die in the next few minutes. Help!" This problem can be solved only by devising a way for panickers to rate the affective impact of their thought or the conviction attached to it independently from how much fear they experienced in association with it.

2. If catastrophic thoughts occur naturally, a PA will follow them. If a PA occurs naturally, catastrophic thoughts will immediately precede or accompany it. Catastrophic thoughts will occur in the context of more enduring catastrophic beliefs.

Co-occurrence of individual thoughts and PAs. If catastrophic thoughts are a sufficient cause of PAs under normal background conditions, a PA should follow every catastrophic thought. Verifying this would require continuous monitoring of thoughts and the ability to judge the potency of each one to cause panic. Psychoanalytic free association is an approximation to continuous monitoring, although the premise of that procedure is that reporting will be incomplete, while judging thought potency is probably impossible in principle, as argued above. If catastrophic thoughts are a necessary cause of PAs, they should be present at the onset of every PA. If such thoughts could be identified as sufficiently potent and present in, say, $95 \%$ of PAs, and if the other $5 \%$ were likely to be errors of measurement, we would be reassured that this hypothesis is correct. Consider then the report of Rachman, Lopatka, and Levitt (1988), who administered a questionnaire asking patients with a diagnosis of PD whether attacks they had just had during exposure to a fearful place or situation had been accompanied by catastrophic thoughts. For $27 \%$ of the PAs the answer was no. Other studies have also looked for catastrophic thoughts or a sense of threat or danger preceding PAs and failed to find them in many attacks (Kenardy, Fried, Kraemer, \& Taylor, 1992; Kenardy \& Taylor, 1999; Zucker et al., 1989). These findings leave proponents of CCT with a hard choice: They must either accept them as legitimate falsifications of their theory and abandon it, or they must question the adequacy of the empirical assessment of the thoughts, opening them to the accusation of entertaining unfalsifiable beliefs. The second choice is not unreasonable, nor is the conclusion that observations of this kind are incapable of falsifying CCT.

Catastrophic beliefs. Transient catastrophic thoughts are likely to arise in the context of more enduring catastrophic beliefs, so the absence of beliefs in PA patients between attacks that anxiety or its symptoms are somehow dangerous would be evidence against CCT. In fact, scores on the Body Sensations Interpretation Questionnaire (Clark et al., 1997) demonstrate that panickers are specifically disposed to misinterpret cardiovascularrespiratory sensations catastrophically. PD patients also have higher scores than controls on other Anxiety Sensitivity questionnaires (for reviews, see Taylor, 1999) such as the Anxiety Sensitivity Index, but this elevation is not specific, since scores are as high in posttraumatic stress disorder and not significantly lower in social phobia (Cox, Borger, \& Enns, 1999). Of course, patients with various diagnoses may have PAs, and at issue here is whether patients who have PAs also have catastrophic beliefs. Available evidence is that they often do, but not always. However, like reports of thoughts, reports of beliefs can be discounted as inadequate or biased, and insofar as these objections are valid, Proposition 2 is unfalsifiable.

Temporal sequence. Although theoretically and empirically questionable, catastrophic cognitions could conceivably be proven to cause PAs by showing that sufficiently potent thoughts precede rather than follow individual attacks, even the patient's first attack. Of course, it is equally conceivable that PAs precede and cause catastrophic thoughts. A reasonable person suddenly, out of the blue, experiencing extreme anxiety and its accompanying bodily symptoms will try to understand their portent. Dire thoughts about 
one's physical and mental health are natural, especially if such attacks had never been experienced before. Subsequent exaggerated attention to, and anxiety about, bodily sensations is hardly irrational. Furthermore, after one or more attacks the panicker may learn that certain feelings and bodily sensations presage attacks and may come to fear them secondarily (Bouton, Mineka, \& Barlow, 2001). In that case, catastrophic thoughts would not be a necessary cause of PAs, preceding or accompanying all natural attacks as expressed in Proposition 2, but might serve to maintain them as a contributing cause, even at times in the absence of the original trigger. This modified version of CCT has less explanatory power than the original if it cannot specify what those original triggers were.

Although attacks come on over many seconds or minutes, the interleaved sequence of thoughts, perceptions, and emotions occurring in individual attacks probably cannot be reliably ascertained through introspection. An interview study of $20 \mathrm{PD}$ patients and 10 controls found that $70 \%$ of panickers reported that catastrophic thoughts followed, rather than preceded, feelings of anxiety (Zucker et al., 1989). Yet it is unlikely that perception of feelings or of bodily sensations can occur without some thought occurring and that early thought might well include an intimation of danger. Anxiety can be expected to be accompanied by dire thoughts. Thus, claims about the sequence of events in individual attacks are unconvincing.

One study, based on interviews concluded that at the first panic experience, in 9 of 10 patients the sequence of events was bodily sensation, followed by panic, followed by catastrophic cognitions (Wolpe \& Rowan, 1988). But answers to the historical question, What thoughts accompanied the initial PAs? are even more unreliable than answers about recent attacks since they are potentially distorted by fading memories revised in light of subsequent events. Thus, reports of early attacks unaccompanied by catastrophic thoughts cannot decisively falsify this proposition. Prospective studies of catastrophic beliefs have attempted to show that people who have not had PAs but who are afraid of their bodily sensations go on to develop PAs later in life (for a review, see Schmidt, 1999). A general limitation of such studies is that fears of bodily sensations may have originated from prior events that, although not remembered or described as PAs, had their essential features.

Nonthinking states of consciousness. Another route toward falsification of this proposition would be to show that natural PAs occur under circumstances where catastrophic thoughts are impossible. The occurrence of PAs in animals incapable of such complex thoughts as catastrophic cognitions would damage the theory, since evolutionary continuity speaks for similar mechanisms in both animals and humans. However, most animal models of PD have involved inducing fear in animals with shocks (Baum, 1986) or chemicals that allegedly provoke panic in humans, and whether fear induced in animals in these ways is really comparable to human panic remains uncertain.

On the other hand, arguments based on PAs in sleeping humans do not depend on similarities across species. About $65 \%$ of patients whose PD can be diagnosed on the basis of waking attacks, report similar attacks waking them out of sleep (Uhde, 2000). It is plausible that sleep attacks are genuine PAs with an identical mechanism to waking attacks, since they occur in the same individuals. Sleep attacks in PD patients have been observed during Stage 2 or slow wave sleep, when unlike REM, thoughts of the complexity of catastrophic ones are seldom reported when the sleeper is awakened, and when body sensations capable of eliciting catastrophic cognitions may not be perceived or assessed as dangerous. Yet these observations are incapable of falsifying CCT because of uncertainly whether sleeping subjects are incapable of entertaining thoughts of sufficient complexity to appraise sensations during sleep as dangerous. Some experiments addressing this issue are mentioned below. On the other hand, reports of catastrophic thoughts on awakening do not prove that such thoughts occurred during sleep and caused awakening; they may first have occurred after the sleepers partially or completely woke up.

3. Catastrophic thoughts are caused naturally by relevant internal or external stimuli.

According to CCT, catastrophic thoughts are a necessary part of a causal chain resulting in a PA, but are not necessarily the first cause in the chain. Thus, an individually specific internal or external stimulus alone or in combination is a sufficient cause of PAs under normal background conditions. For example, fear of an imminent heart attack may be precipitated by cardiac perceptions originating from benign physical activity but which are suddenly interpreted as harbingers of death. Reading a newspaper article about heart attacks might bring catastrophic thoughts into play via a semantic route. Falsifying that such internal or external stimuli are precursors of catastrophic thoughts would follow the same logic as falsifying that catastrophic thoughts are precursors of PAs: If the purported causes fail to precede or at least accompany catastrophic thoughts, Proposition 3 must be false. The apparent spontaneity or unexpectedness of certain PAs, however, is a sign that any eliciting stimuli must be subtle and difficult to detect. In the case of external stimuli, if the panicker usually cannot identify the stimulus, it is unlikely that an outside observer of the panicker in the situation could do better. Internal, somatic stimuli might be documented physiologically, but since these are the same that occur in anxiety, distinguishing whether such stimuli are a cause or effect of panic anxiety, is laden with the same uncertainties of sequencing rapid and overlapping processes discussed for catastrophic thoughts as precursors of PAs.

4. If catastrophic thoughts are induced, a PA will follow. If a PA is induced, catastrophic thoughts will have preceded or accompanied it.

Ehlers, Margraf, Roth, Taylor, and Birbaumer (1988) produced self-report and physiological evidence of anxiety in PD patients by giving them false feedback that their heart rates were rising precipitously. One of the 25 patients tested volunteered that she had had a PA, showing that information alone is sometimes able to trigger attacks, presumably through induction of catastrophic thoughts. Clark and others (cited in Clark, 1996) reported anecdotally that panic patients had PAs while reading pairs of words epitomizing catastrophic thoughts, such as palpitations-dying. "Biological" provocations may actually induce PAs by the information imparted to subjects in obtaining their informed consent. Implicitly or explicitly this information inevitably conveys to previous panickers a new, rational catastrophic thought specifically related to the test: "The experimenters have devised something that they think will make panickers panic. I have panicked 
before, so if they are competent, I will be made to panic by their procedure." Informational induction of PAs or the efficacy of psychotherapies in alleviating PAs are demonstrations that changing thoughts is one route to inducing feelings, in other words, that thoughts are a sufficient cause of panic. However, as discussed in more detail below with respect to informational reduction of PAs, this does not mean that catastrophic thoughts are the necessary, root cause of natural PAs. Proposition 4 could be falsified if it could be shown that a catastrophic thought had been informationally induced but no PA ensued, but that thought would have to be known a priori to possess content and potency adequate for precipitating a PA, and that cannot be established unequivocally.

After the establishment of the PD diagnosis there was a boom in research attempting to find substances that could provoke PAs, which had as its goal the discovery of biological as opposed to psychological causes of panic. Noncognitive interpretations of results of these experiments were challenged by Margraf, Ehlers, and Roth (1986) and later by many others, who noted that successful provocations tended to induce sensations frightening to PD patients, which could be presumed to induce catastrophic cognitions. Since these cognitions might be expected to arise from attitudes represented in the Anxiety Sensitivity Index, it is consistent with the suggestion of Margraf et al. that, at least in individuals without PD, scores on this questionnaire are a predictor of anxiety responses to HV or to carbon dioxide inhalation (Stein \& Rapee, 1999). However, in one study using lactate infusions, such cognitions were denied by most of the patients who had PAs (Aronson, Whitaker-Azmitia, \& Caraseti, 1989), but as usual the question arises about whether these denials can be taken at face value.

One route to falsifying Proposition 4 might be to find two substances that induce identical sensations or no sensations, which would then be equal in inducing catastrophic thoughts, but which turned out to be unequal in inducing PAs. Of course, for such a test to be valid, the evoked PAs would have to be the same as natural attacks in symptoms and time course, and not simply a doseproportional effect of an anxiogenic substance with a gradual onset. Unfortunately all existing provocations, such as lactate, HV, inhaled carbon dioxide, caffeine, yohimbine, and doxapram elicit distinguishable sensations even after the best dose-matching, making it possible that the sensations of the more potent substance were simply more potent for inducing catastrophic thoughts. A priori matching of the potency of substance-induced symptoms would be uncertain. Flumazenil, a blocker of benzodiazepines, apparently was able to produce PAs in PD patients in the absence of any sensations (Nutt, Glue, Lawson, \& Wilson, 1990), which could circumvent these objections, but in an attempted replication it failed to produce PAs in a similar population (Ströhle, Kellner, Holsboer, \& Wiedemann, 1999).

Note that even if some biological substance could be shown to induce PAs without catastrophic thoughts, we could not conclude that the mechanism of induction was a simple chemical one not involving learning. The substance might work by inducing sensations associated with past PAs that, through a process of emotional conditioning operating independently of propositional learning (Bouton et al., 2001), would be able to trigger PAs.

Another route to falsifying Proposition 4 might be to show that panic provocations are effective even when the state of the subject's mind is such that it cannot entertain thoughts as complex as catastrophic ones. Induction of PAs during sleep seemed to be a route to such a falsification, but this would have no advantage over waking provocations if fearful expectations could influence the processing of danger signals even during sleep, which is what Craske and Freed (1995) have attempted to show. They found that PD patients reported less anxiety after being awakened from sleep by tone signals that supposedly indicated autonomic arousal when the patients had been reassured before falling asleep that the signaled arousal was safe. A methodologically improved version of this experiment showed a similar result (Craske et al., 2002). Although the experimenters interpreted their finding as indicating that danger attribution of bodily sensations could occur during sleep, they could not exclude the possibility that these attributions were made after the subjects were partially or fully awake.

5. If a catastrophic thought is suppressed, a PA will be suppressed. If a PA is suppressed, catastrophic thoughts preceding it will have been suppressed.

CBTs of PD are designed to modify and attenuate catastrophic thoughts by communicating the therapy rationale, by cognitive restructuring, and by interoceptive exposure (Barlow, 1997), all of which serve to combat the idea that panic is dangerous. Of course, as far as this proposition is concerned, it is irrelevant whether CBT reduces catastrophic thoughts through its specific procedures. The proposition would be equally supported if panic frequency was reduced in step with reductions in catastrophic thinking by therapies based on relaxation, meditation, animal magnetism, eye movement desensitization, Christian Science, or the power of positive thinking (see the introduction to W. T. Roth \& Yalom, 1997).

Empirical studies confirm that CBT indeed lowers both panic frequency and client reports of catastrophic thoughts and beliefs (Otto \& Reilly-Harrington, 1999). Patients with persisting catastrophic beliefs after a treatment that rendered them temporarily panic free are the most likely to relapse (Clark et al., 1994). Of course, had these beliefs been strong enough to give rise to catastrophic thoughts during the panic-free period, this proposition would have been falsified. Not only are spontaneous PAs reduced by cognitive interventions, but consistent with CCT, PAs to provocations such as doxapram (Abelson, Nesse, Weg, \& Curtis, 1996) and carbon dioxide inhalation (Schmidt, Trakowski, \& Staab, 1997) also become less likely, presumably in proportion to reductions in catastrophic thinking.

Falsifying this proposition would require the demonstration that a thought whose potency and content was sufficient to produce PAs occurred without an attack, which would require accurate, continuous (over days and weeks) monitoring and reporting of the stream of consciousness and an ability to assess the emotional impact of cold cognitions, neither of which is feasible. Less directly, beliefs presumably leading to such thoughts could be shown to persist in spite of effective therapy, but difficulties in quantifying beliefs and the likelihood of individual exceptions make this route to falsification uncertain. Perhaps one of the supposedly noncognitive psychotherapies could be shown to suppress PAs without reducing catastrophic thinking, but if catastrophic thinking is an inevitable aspect of panic anxiety this will not succeed. 
On a more general level, the fact that cognitive therapies can reduce panic at all is regarded as evidence that at least some kind of thoughts cause PD. However, as important as this fact is practically, it does not mean that thoughts are necessary, root causes of panic. If we assume that the emotion panic is a complex of thoughts, behaviors, and somatic reactions, that complex will necessarily be altered if any of its elements is altered. Perhaps the panic complex originates from a yet-to-be-elucidated brain event, and even without many, or even any, repetitions of this event, the complex lives on like a traumatic memory in posttraumatic stress disorder, which is reactivated in flashbacks. Explanations have been advanced for why such phenomena fail to extinguish long after the traumatic event (Brewin, 2001; Ehlers \& Clark, 2000). But even if this hypothetical brain event continues to accompany PAs, because it soon establishes itself as part of a cognitivesomatic-behavioral complex, modifying any part of this complex might alter the subject's experience. Remitted panic patients sometimes report that they still periodically experience "funny feelings" but that these feelings are no longer associated with thoughts of disaster or impulses to run.

Pharmacological therapies that reduce PAs should reduce catastrophic thinking. We could falsify Proposition 5 by demonstrating that a drug can reduce panic without doing away with such thinking. Like provocations, anxiolytic drugs cannot be assumed to act noncognitively, since even in placebo-controlled efficacy trials, the sensations they produce can influence thoughts. It is reasonable to expect that an active drug but not a placebo will be beneficial while being taken (but not when discontinued), and the conviction that the drug is active is strengthened by awareness of its side effects. Klein and Klein (1989) argued that the racing heart produced by the anticholinergic side effects of the antipanic drug imipramine contradicted CCT because an anxiety-related kind of bodily sensation was being amplified. However, a racing heart may paradoxically be reassuring to the panic patient, who interprets it as a sign of safety rather than threat. The catastrophic thought "My heart is racing; I may die" may be transformed to "My heart is racing; I am protected." For drugs without this side effect or even without any side effect, the thought may become "If my heart raced for no reason, I might die, but now my heart will be normal since I am taking this drug."

Eliminating the potentially confounding role of somatic sensations raises the same problems as for chemical panic provocations. Specific pharmacological actions would be most convincing when two drugs eliciting identical changes in body sensations, or two drugs eliciting no changes at all, differ in efficacy. However, such matching is difficult, if not impossible, to achieve. On the other hand, it should be possible to find a drug whose side effects are greater than another drug but less efficacious, or drugs whose side effects have a different time course than their antipanic effect. In the case of tricycle antidepressants, for example, anticholinergic side effects are immediately apparent, while the antipanic and antidepressant effects are delayed.

\section{Heuristic Value}

This theory is comprehensive in including thinking, basic cognitive processes such as perception and attention, and somatic events into a wide web of explanation. In terms of the phenomena of PAs, it explicitly addresses the predisposing and precipitating causes of attacks, but oddly does not tackle the question of why individual PAs end. If they end because of a change in thinking, why is that change so impermanent that attacks recur after a few days or weeks? Consistent, continuing failure to learn that negative health expectations fail to materialize seems to require denial of psychotic proportions. Subtle avoidance behaviors and an irrational belief that good luck will be followed by bad have been proposed to explain this resistance to giving up catastrophic beliefs (McNally, 1999).

The heuristic value of CCT was evident in its ability to challenge the emerging consensus among mental health professionals that PAs required a biological explanation instead of a psychological one. A controversial theory, it has stimulated numerous clinical and nonclinical research studies and publications. In addition, it proposed a new, plausible psychological explanation that became the basis of a distinctive, new psychological therapy whose efficacy was also a surprise to those who had believed that PD could only be treated with drugs.

\section{VCT}

\section{Core Thesis}

This theory usually appears as a component of other theories rather than as a comprehensive explanation of PAs, but because evidence regarding it is distinct from evidence for other theory components, we consider it separately here. Its core thesis is that the PA's characteristic rapid escalation to high anxiety levels is caused by a positive feedback loop between anxiety and the effects of anxiety. This loop produces an explosive amplification of anxiety on a time scale of between tens of seconds and a few minutes. Typical examples of anxiety effects given as being fed back are heart pounding and racing, trembling, or HV's lightheadedness and finger tingling, but more central manifestations of anxiety such as depersonalization or derealization have also been mentioned. Some versions of the theory consider the fear of anxiety effects to have been learned, perhaps as a result of earlier PAs (Bouton et al., 2001), while others consider at least shortness of breath, a feeling often accompanying anxiety, to be intrinsically unpleasant and frightening (Ley, 1989). Thus, versions differ in how much cognitive appraisal contributes to the escalating anxiety.

CCT usually incorporates VCT, both in its theoretical descriptions (Clark, 1986) and in the therapeutic rationales based on CCT that are conveyed to the patient (Wilhelm \& Margraf, 1997). The HVT of panic has been supplemented with VCT (Ley, 1985a). VCT would fit well with the SFAT since anxiety increases the feeling of not getting enough air. However, VCT antedates all of these theories, having entered the scene long before PD appeared on center stage, which is hardly surprising considering its close kinship to the classic James-Lange theory of emotion. Tracing the history of this idea, Alpers, Wilhelm, and Roth (2001) found VCT expressed in a startlingly modern way in 1937 :

Anyone may have an attack of palpitations on excitement, ... in neurosis it seems to come on when the patient is undisturbed. . . . The reaction may be prolonged because for some reason it is dreaded either because it is so uncomfortable in itself or because of the consequences it is supposed to bring about. . . . fear causes palpitations... . the more palpitations he gets, the greater will his fear become. There need be no end to their vicious circle unless his views are changed. (Ross, 1937, pp. 23-24) 
Tests

The requirements for falsification of VCT differ according to what kinds of anxiety symptoms are considered eligible for feedback, what level of awareness the perception of anxiety symptoms is believed to require, and how complex their assessment is postulated to be. Conceivably testable propositions derived from the theory are detailed below.

1. The occurrence of a manifestation of anxiety postulated to be a stimulus for anxiety can be precisely and objectively established independent of a PA.

A feedback loop requires that the input and output of the mechanism can be distinguished, such as when there is a time delay between them. Postulating a pure fear-of-fear feedback loop in which fear leads to more fear leads to more fear, and so forth, would be meaningless, because it would simply be another way of saying that fear increases. However, if a manifestation of anxiety can be manipulated independently of other manifestations of anxiety, a separation between input and output has been achieved. This kind of separation was an assumption of the James-Lange theory of emotion, where motor and autonomic behavior were the causes of an emotional feeling, but is not assumed by modern theories where cognitive, behavioral, and physiological elements of an emotional event are considered part of a single network. Without an empirically convincing separation, VCT is unfalsifiable; with a separation, a causal analysis is possible. The independence of input and output must be demonstrated by showing that each can vary without the other varying. Heart rate, for example, can easily be raised independently from panic by physical activity, but in some people, heart rate may always be elevated when panic is reported. However, the second kind of dissociation can be achieved with beta-blockers.

2. If a PA occurs naturally, some manifestation of anxiety satisfying Proposition 1 increases simultaneously with, or within seconds before, anxiety increases. For the CCT version of this theory to be true, the panicker must be sufficiently aware of the manifestation of anxiety and cognitively acute enough, to assess its portent as catastrophic. For an HV version involving CCT to be true, the manifestation must be one that can be produced by $\mathrm{HV}$.

If the conditions of the previous proposition are met, manifestations of anxiety could be monitored during natural PAs, and the co-occurrence and the timing of, say, heart rate increases and other independent evidence of a PA could be evaluated. Certain quantitative relationships between output and input elements permitting a positive feedback loop leading to rapid spirals might be established. However, the truth of Proposition 2 can be falsified convincingly only for individual anxiety output elements. We are doubtful if general falsification of VCT can never be achieved in practice, since every reported and unreported manifestation or any combination of them could be the critical input for the feedback loop.

VCT could be valid as a mechanism for escalation of anxiety even if HVT and CCT are false. The element fed back may have nothing to do with HV and may involve automatic, unconscious somatic anxiety responses through Pavlovian visceral condition- ing, which has been demonstrated in animals incapable of catastrophic thinking of a human sort (Razran, 1961). Bouton et al. (2001) give examples of conditioning experiments where the onset of an event signals the rest of the event or a small dose of a drug signals a higher dose. They find it plausible to extrapolate from the examples cited by Razran (1961) to visceral signs associated with minimal anxiety becoming conditioned to produce severe anxiety. For interoceptive conditioning to be relevant to VCT, it is only necessary that the conditions of Proposition 1 to be met for one somatic element and that an increase in that element be linked to an increase in anxiety defined independently of that element. Showing that people with previous PAs react to unnoticed changes in heart rate or breathing with subsequent increases in self-reported anxiety, or even in another somatic output element such as skin conductance, would be a step in making these assumptions plausible. Of course, this scenario does not explain the first attack or attacks, which are needed as URs that are elicited by some other mechanism until conditioning has had a chance to take place. (For a suggestion about the first attack, see Jacobs \& Nadel, 1999.) The theoretical importance of interoceptive conditioning extends beyond VCT since it can explain PAs after the first ones without recourse to an escalation of anxiety. Yet, its inability to explain the first attacks limits it to being a theory of the continuing occurrence of PAs rather than their primary cause.

VCT could be valid as a mechanism for escalation of anxiety even if HVT and CCT are false. The element fed back may have nothing to do with $\mathrm{HV}$, and may involve automatic, unconscious somatic anxiety responses through Pavlovian visceral conditioning, which has been demonstrated in animals incapable of catastrophic thinking (Razran, 1961). A number of theorists have extrapolated from these animal experiments to human PAs, postulating that in panickers visceral sensations have become conditioned stimuli for fear (Barlow, 1988; Goldstein \& Chambless, 1978; Wolpe \& Rowan, 1988). Such conditioning may be unaccompanied by thoughts, catastrophic or otherwise, because aversive emotional learning can occur in humans without giving rise to propositional, declarative knowledge about it (Bouton et al., 2001).

For the CCT version of VCT, the panicker must be sufficiently aware of the manifestation of anxiety and cognitively acute enough to realize its catastrophic portent. Whether body changes are perceptible can be approached experimentally. In the nomenclature of signal detection theory, the perceiver must report the body change under investigation when it occurs (a failure to do so is a "miss") but must not report a change when it has not occurred (doing so is a "false alarm"). Ehlers has studied extensively the perception of heartbeat in PD patients (Ehlers \& Breuer, 1996) and has confirmed that such perception is possible, although not very accurate. Men are better heart rate perceivers than women, inconsistent with the higher incidence of women panickers. Other cardiovascular measures such as stroke volume or pulse pressure may be easier than heart rate to perceive (for a review, see Vaitl, 1996). Symptoms such as trembling, fast breathing, and sweating are perceptible even to external observers. The main barrier to selfperception of these symptoms would be inattention.

For the HV version of VCT, the manifestation must be one that can be produced by HV. In one experiment, patients supposedly suffering from an $\mathrm{HV}$ syndrome breathed through a mask from a reservoir whose $\mathrm{CO}_{2}$ level could be controlled (Hornsveld, Garssen, Fiedeldij Dop, van Spiegel, \& de Haes, 1996). When patients 
hyperventilated, adding $\mathrm{CO}_{2}$ to the reservoir could prevent falls in their $\mathrm{CO}_{2}$ levels. Many patients reported symptoms of $\mathrm{HV}$ even when their $\mathrm{pCO}_{2}$ levels were kept normocapnic. Thus, these reports were false alarms. However, even though the defining feature of $\mathrm{HV}$ is hypocapnia, another feature, such as fast breathing, could be the one that the panicker perceives and fears, perhaps by prior conditioning. At a cognitive level, a link between fast breathing and panic may have been forged by prior information given to the patients about HV syndrome. Furthermore, patients to whom this syndrome is attributed may be fearful about bodily functions and sensations in general, fast breathing being only the easily perceptible example that happened to be under scrutiny in this experiment.

3. If a certain manifestation of anxiety is induced, a PA will follow. If a PA is induced, that manifestation of anxiety will have preceded or accompanied it.

In practice it is easy to induce somatic manifestations of anxiety controlled by the autonomic nervous system. Heart racing can be produced by exercise or by cholinergic blockers. A feeling of dyspnea can be induced by inhaled gasses. Many if not all of the PA provocations induce somatic symptoms of anxiety, which according to CCT is how these provocations work. The stumbling block to general falsification of this proposition as for the previous proposition is that the set of possible somatic manifestations is large and poorly delimited. If an experimenter succeeds in showing, for example, that exercise or drug-induced increases in heart rate do not induce anxiety in a given panicker, the possibility remains that some other somatic manifestation is the critical one, or that a certain combination is required. Thus, the theory is too poorly specified to be disconfirmed.

4. If a relevant manifestation of anxiety is suppressed, a PA will be suppressed. If a PA is suppressed, the relevant manifestation of anxiety preceding it will have been suppressed.

Beta-blocking drugs can suppress cardiovascular manifestations such as heart racing or pounding. The demonstrated failure of beta-blockers as antipanic drugs in placebo-controlled trials effectively falsifies VCT for cardiac sensations (Munjack et al., 1989), but other physiological systems linked to anxiety remain unblocked by these drugs. Voluntary suppression of symptoms such as $\mathrm{HV}$ or muscle tension or biofeedback-trained suppression of involuntary ANS symptoms might halt attacks if VCT is true. However, general falsification of VCT by this route is hardly feasible since it would require the suppression of all conceivable anxiety manifestations before concluding that the continuing occurrence of PAs was incompatible with the truth of VCT.

\section{Heuristic Value}

VCT is able to explain only how anxiety can rapidly escalate in the absence of increasingly threatening external stimuli and does not tackle the question of how certain body sensations became feared or why individual PAs end. In spite of these limitations, VCT has been compelling for theoreticians and for clinicians, whose patients apparently accept it easily as a therapeutic rationale. The theory challenges the common belief, whether true or false, that sensations like a pounding heart simply go along with being afraid rather than helping to cause the fear. Recent research relating interoception to a variety of medical and psychological problems (Vaitl, 1996) has been stimulated by VCT.

\section{TAT}

\section{Core Thesis}

Over the last decades Barlow and his colleagues (Barlow, 1988, 2002; Bouton et al., 2001) have developed a theoretical framework for understanding PAs and PD. It postulates three kinds of alarms during which anxiety increases (true, false, and learned) and two anxiety systems (an innate primitive present-oriented fear system and a future-oriented defensive anxiety system). Although the immediate trigger for individual false alarms is not specified, probability of an alarm is increased by stressors, by early experiences, by genetic influences on the fear mechanism, and by higher tonic anxiety levels. These elements constitute a "stress-diathesis model" of panic. False fear alarms are not infrequent in the general population and usually do not lead to diagnosable PD, because most people who experience them never develop a persistent concern about additional attacks, worry about the implications of the attacks, or show a significant change in behavior. Development of these additional features depends on the individual's diathesis toward anxiety and learned alarms, which is determined by a distinct set of biological and psychological factors. PAs can be of two types: spontaneous firing of the fear system in the absence of danger cues (false alarms) and later in the course of the disorder, conditioned evocation by interoceptive or exteroceptive cues of the fear system or of the anxiety system (both learned alarms). In other words, there can be two kinds of learned PAs based on fear or anxiety systems. In the latter case, a "brief burst of discrete conditioned anxiety" (Barlow, 2002, p. 244) is elicited by interoceptive cues.

Some of these ideas overlap with those of other more specific theories that we discuss in this article. Klein's SFAT also postulates separate panic and anxiety systems but, unlike TAT, identifies false alarms exclusively with suffocation-related mechanisms. Like TAT, Klein considers generalized apprehension after PAs and agoraphobia to be examples of learned anxious anticipation of future PAs. The HVT of panic as elaborated by Ley (1992b) also proposes the existence of several kinds of PA in addition to the basic HV type. Gorman's NH follows Klein in assuming the existence of separate panic and anxiety systems and specifies their pathways in the brain. It is even arguable that counting TAT as a theory of PAs is incorrect, since its main concern is to explain the development of PD by conditioning. Yet, TAT does make statements about the phenomenon of panic that warrants its inclusion here. We concentrate here on its statements most relevant to that phenomenon, neglecting in this short review its weaving together of findings from a vast literature into a comprehensive developmental theory of PD.

\section{Tests}

TAT aspires to a better understanding of anxiety and how it is triggered by analyzing it into categories with distinct features. It often reads like a descriptive summary of research findings, sticking close to the facts. Yet, as a useful theory should, it contains 
propositions that extend beyond the facts, and thus questioning their testability is advisable. Here we apply the concept of falsifiability to the categories that this theory postulates. If the hypothetical categories are meaningful, they should be able to exclude unequivocally individual anxiety episodes from that category.

1. An episode of experienced anxiety can be categorized according to what kind of alarm it is: false, true, or learned.

To be able to disqualify an episode as a true alarm, researchers would need to know that it occurred in the absence of environmental stimuli known to trigger such alarms, such as an angry face, a creature slithering toward a person, loud noises, or rising $\mathrm{CO}_{2}$. External stimuli that cause marked anxiety can probably be accurately reported by the person experiencing the anxiety, and in principle, such reports could be confirmed by outside observers. On the other hand, if internal stimuli are able to produce true alarms, for example, rising arterial $\mathrm{CO}_{2}$, then the person experiencing the alarm might not be able to distinguish this alarm from a false alarm. Physical measuring devices, however, could confirm that $\mathrm{CO}_{2}$ was rising. Little is known about whether there are other intrinsically frightening internal stimuli.

The category of learned alarms presents greater falsification difficulties. Can we ever be sure that an anxiety episode was not triggered by something learned? It has often been argued that what seems to be a true alarm may actually have been learned, or vice versa. Children learn early that angry faces mean trouble. They learn from experience or from people in their environment that insects can sting. Emotional learning can be both over- and underreported. When a woman tells us that she used not to have PAs at the cinema until she once had a PA there, we feel certain that her subsequent anxiety when entering a theater is a learned alarm. Yet, we may not believe the denials of agoraphobics that any frightening past conditioning event or PA preceded their current situational fears. Many people with phobias recall no traumatic conditioning events. For agoraphobic stimuli that are not evolutionarily prepared, such as driving an automobile or flying in an airplane, past conditioning events such as accidents, turbulence, or PAs would be expected to have occurred before the onset of the avoidance, yet such a memory is often missing (Poulton \& Menzies, 2002). Perhaps in those phobias, false alarms did occur on one or more occasions but were perceived as an integral part of a fear reaction to the situation rather than as separate conditioning events. In any case, reports of learning or not learning are untrustworthy. Sensitization can be mistaken for learning, and learning can take place without its being noticed or verbalizable.

The category of false alarm is the most problematic from the standpoint of falsifiability because it is defined by the absence of the two possible alternatives, a true alarm or a learned alarm. The gamut of potential threats is wide and subtle, including fleeting, unconscious thoughts, environmental features that an outside observer without attentional threat biases could easily miss, and private bodily sensations. When attempting to classify an individual PA, the researcher can never be certain that none of these threats was present. Neither self-report, nor outside observation, nor physiological monitoring are completely reliable, so a spurious residual category of apparently non-threat-related PAs is bound to exist. The goal of the experimenter must be to find triggers for supposed false alarms, say, epileptiform discharges in a certain part of the brain, rather than to be content with an equivocal exclusion category.

2. An episode of experienced anxiety in humans can be categorized according to the contributions of two underlying biological systems, fear and anxiety.

From the standpoint of falsifiability, we need to ask whether we could conceivably demonstrate the existence of only one anxiety system or of more than two systems. Since as far as is known, PAs are a human phenomenon, a demonstration of two anxiety systems in animals would only indirectly support this proposition. Human evidence for separate fear and anxiety systems based on structural equation modeling of symptoms (Brown, Chorpita, \& Barlow, 1998; Joiner et al., 1999) confirm that in patients, anxiety of the acute, unexpected PA type is statistically distinct from more sustained anxiety. To a certain extent this could have been a result of the patient sample being recruited selectively from specific $D S M-I V$ categories. Generalized anxiety disorder patients are the prototype of subacute future-oriented anxiety, and PD patients and specific phobic patients, the prototypes of immediate fear. However, self-reported distinctions such as abruptness or whether the fear is present or future oriented are unlikely to ever be reliable enough to establish a dichotomy of types, especially in population samples who have not passed through a categorical diagnostic sieve. For example, people who have experienced an anxiety episode often cannot precisely specify the amount of time from the initial onset to the peak of the attack. During episodes, both immediate and future concerns may be recognizable: The fear of immediate medical collapse can coexist with longer term fears of the implications of demonstrating an embarrassing "weakness" in front of other people or of causing an automobile accident.

Thus, biological distinctions between types of anxiety are more likely to be convincing than a self-reported one, especially since the two systems are postulated to be biologically separate. For example, there is evidence that "anticipatory anxiety" is associated with cortisol responses while panic anxiety is not (Sinha et al., 1999). To falsify the proposition that two anxiety systems exist, we might collect evidence for a single system by showing that neither cortisol nor any other biological indicator varies for any other reasons than anxiety intensity or individual differences unrelated to emotion. However, since there are many potential indicators, this proposition is unlikely to be falsified by exhausting the possibilities.

\section{Heuristic Value}

The main value of TAT is heuristic, inviting researchers to test more specific versions of it. In fact, the future may bring demonstrations of considerately more than two anxiety systems or systems related to various aspects of anxiety-from orientation and exploratory behavior in response to distant threats, to the emergency fight-flight response to immediate threats. The theoretical writings of the writers who explicated TAT have encouraged the application of learning theory to understanding panic. They explained how the same physiological event, an increase in heart rate, albeit of different intensities could be both an conditioned stimulus and CR, using as examples where the onset of an event signals the rest of the event or a small dose of a drug signals a 
higher dose (Bouton et al., 2001). They argued that it is plausible to extrapolate from the examples cited by Razran (1961) to visceral signs associated with minimal anxiety becoming conditioned to produce severe anxiety. That interoceptive cues can trigger panic has been a rationale for developing an interoceptive exposure component in treatment packages of PD.

\section{HVT}

\section{Core Thesis}

The basic tenet of HVT is that there is a correspondence between acute decreases in arterial $\mathrm{pCO}_{2}$ (hypocapnia with respect to resting levels), the defining characteristic of $\mathrm{HV}$, and acute anxious states. In addition, most proponents believe that the hypocapnia causes the anxiety, although a few have regarded HV as simply a manifestation of anxiety in certain people (for a review, see Hardonk \& Beumer, 1979). Historically, HV has been linked to several psychiatric disorders, some far predating PD (Hardonk \& Beumer, 1979). In 1929 HV was advanced to explain DaCosta's, or "irritable heart," syndrome, which incapacitated soldiers in the American Civil War. In 1938 HV was proposed to lie behind "neurocirculatory asthenia" or "effort syndrome." Shortly after the diagnosis of PD became official, Ley (1985b) proposed that acute HV caused PAs. He elaborated his HV theory of PAs to include additional elements: a vicious circle mechanism (Ley, 1985a); a lowered threshold for panic due to chronic HV (Ley, 1987); an emphasis on the automatic fear-eliciting nature of involuntary dyspnea (Ley, 1989); a hypoxic origin for cognitive panic symptoms (Ley, 1989); and a classification of PAs, two kinds of which are not caused by HV (Ley, 1992b). A different classification strategy, beginning in 1937, had been to define a "hyperventilation syndrome" distinct from other psychiatric illnesses, but the basis for a variety of "neurotic" symptoms.

\section{Tests}

The falsifiability of the basic HV theory of PAs hinges on the feasibility of tests like the ones below and whether their results can be interpreted unambiguously.

1. The occurrence of HV can be precisely and objectively established independent of a PA.

HV can be precisely defined in terms of a decrease in normal values of arterial $\mathrm{pCO}_{2}$. Because $\mathrm{HV}$ refers to respiration, a definition in terms of respiration rate, tidal volume (the volume of air moved from the peak of expiration to the depth of inspiration), or minute volume (the volume of air breathed per minute $=$ respiratory rate $\times$ tidal volume) is also possible, but these variables are probably less directly related to the bodily changes believed to cause the symptoms of HV. Arterial pH might be even more closely related to those bodily changes, but it is inconvenient to measure, while arterial $\mathrm{pCO}_{2}$ can be estimated accurately and noninvasively from end-tidal $\mathrm{pCO}_{2}$ in the expired breath (the highest $\mathrm{pCO}_{2}$ level coming from air deep in the lungs, which occurs near the end of expiration). Since the emotion of anxiety includes physiological changes as an integral component, it is conceivable that HV could be construed as inseparable from anxiety, precluding causal relationships. That this is not the case is proved by experiments that show other features of anxiety and HV to be quantitatively or temporally dissociable, the same experiments that are relevant for proving causal relationships (Garssen, Buikhuisen, \& van Dyck, 1996; Hibbert \& Pilsbury, 1989). Thus, Proposition 1 is correct.

2. If a PA occurs naturally, HV will precede or accompany it. If HV occurs naturally, a PA will follow it.

A recording device has been invented that records $\mathrm{pCO}_{2}$ levels in skin blood vessels transcutaneously, registering the drop in $\mathrm{pCO}_{2}$ produced by voluntary $\mathrm{HV}$ after about $30-90 \mathrm{~s}$. Hibbert and Pilsbury (1989) found distinct drops in $\mathrm{pCO}_{2}$ in 7 of 15 patients who had spontaneous PAs during the recording period. However, Garssen et al. (1996) found drops in only 1 of 24 natural PAs experienced by 14 of $28 \mathrm{PD}$ patients who were monitored. Although the transcutaneous method is limited in its ability to detect small or transient episodes of $\mathrm{HV}$, these studies show that many if not most PAs are not accompanied by substantial HV. Since hypocapnia is the defining characteristic of $\mathrm{HV}$, the negative findings of Garssen et al. have falsified this proposition. A more sensitive device that measures end-tidal $\mathrm{pCO}_{2}$ at the nostrils is able to detect smaller and more transitory HV episodes (Wilhelm, Alpers, Meuret, \& Roth, 2001), such as those following individual sighs, but such episodes are probably too minor and too common to be elicitors of clinical panic. If the basic theory is hedged by asserting that HV explains PAs only in a subset of panickers or in a subset of attacks of individual panickers, we must ask whether that subset is large enough to be important and whether it can it be identified in any other way than a failure to hyperventilate during PAs.

Even if it were demonstrated that HV accompanied PAs, HV might only be part of an anxiety or "fight or flight" response triggered in some other way than by HV. If it could be established that $\mathrm{HV}$ follows rather than precedes panic, a causal relationship could be falsified. Ley (1985a) interviewed 10 agoraphobics, 9 of whom had PAs and asked them whether they had noticed any of the $12 D S M-I V$ symptoms defining a PA during the attack and whether the symptom occurred before or after they experienced panic fear. Eight of the 9 panickers placed the predominance of the symptoms before the fear (an exception was "trembling or shaking"). Since all of these symptoms can occur during voluntary HV, he felt that this sequence indicated that $\mathrm{HV}$ caused the fear. However, even assuming that $\mathrm{HV}$ did produce the symptoms, which is unlikely in light of the findings of Garssen et al. (1996), and assuming also that experimenter expectations did not bias subject reports, self-reporting of a sequence of experiences seems incapable of verifying causal precedence. Since people with PAs quickly grow to fear any indications that an attack is coming on, it is implausible that they would perceive a symptom of previous attacks without immediately being fearful. Furthermore, attention to symptoms and feelings is selective. People may at first be unaware that they are breathing abnormally and they may at first be unaware that they are starting to be anxious. A somatic symptom of anxiety could first draw their attention to the fact that they were becoming anxious. Thus, self-reports of sequences of symptoms and anxiety are too uncertain to verify or falsify a causal claim. 
3. If $\mathrm{HV}$ is induced, a PA will follow. If a PA is induced, HV will have preceded or accompanied it.

The first statement of this proposition appears to have been falsified. In one study in which PAs were objectively assessed and end-tidal $\mathrm{pCO}_{2}$ was monitored, voluntary $\mathrm{HV}$ to a $\mathrm{pCO}_{2}$ of 25 $\mathrm{mmHg}$ for up to $15 \mathrm{~min}$ failed to produce PAs in the majority of people who had previously had such attacks (Gorman et al., 1988). In another study, $8 \mathrm{~min}$ of voluntary $\mathrm{HV}$ at $20 \mathrm{mmHg}$ produced PAs in $58 \%$ of PD patients (Maddock \& Carter, 1991). In a third study, 3 min of voluntary $\mathrm{HV}$ at $20 \mathrm{mmHg}$ (after 6 repeated 1-min $\mathrm{HV}$ and 1-min recovery periods) produced PAs in $36 \%$ of PD patients but 54\% of social phobics (Wilhelm, Gerlach, \& Roth, 2001). Thus, at lower $\mathrm{pCO}_{2}$ levels and with greater respiratory effort than is common in natural attacks, some PD patients do panic, but most natural PAs must be triggered by something other than HV. A fallback position for proponents of HVT is that a sense of control in voluntary HV prevents anxiety from reaching the heights attained when $\mathrm{HV}$ is unconscious and symptoms appear unexpectedly. However, this complicates the theory by the addition of a complex cognitive element, spoiling its original parsimony.

HVT often assumes that acute HV will increase anxiety in most people and that the difference between panickers and nonpanickers is whether or how much they hyperventilate. In fact, anxious patients, regardless of whether they have PAs, tend to respond to HV with more anxiety than controls. In an experiment by Margraf, Ehlers, Herber, Meisner, and Wrobel (1991), reactions to 2 min of voluntary HV were compared for three groups: students who had experienced PAs; socially anxious university students, those whose anxiety had not expressed itself in PAs; and nonanxious control students. Increases in anxiety and anxiety symptoms were greater for both anxious groups than for controls, but the panickers did not react more than the socially anxious. Wilhelm, Gerlach, and Roth (2001) have found similar results in PD and social anxiety disorder patients. In controls, voluntary HV even can elicit positive emotions (Clark \& Hemsley, 1982).

In most experiments where PAs were induced, respiratory measures were not recorded. However, it has been documented that HV may accompany lactate-induced PAs and bicarbonate-induced PAs (Gorman et al., 1989) and epinephrine-induced PAs (van Zijderveld, Veltman, van Dyck, \& van Doornen, 1999), which is a verification of the second statement of this proposition for at least these panicogenic substances. Respiratory stimulants such as doxapram and cholestokinin can produce panic, although it is not clear if HV is the causal factor (Abelson \& Nesse, 1994; Abelson et al., 1996). On the other hand, PAs can be induced by inhaling higherthan-normal concentrations of $\mathrm{CO}_{2}$, in which cases end-tidal $\mathrm{pCO}_{2}$ rises above baseline levels (Papp et al., 1997). This contradicts the second part of Proposition 3, and shows that it is falsifiable.

4. If HV is suppressed, a PA will be suppressed. If a PA is suppressed, HVs preceding it will have been suppressed.

If HVT is true, successful prevention of HV is necessary and sufficient under normal background conditions for eliminating future attacks. The theory would be falsified if patients failed to stop having PAs when their breathing had been normalized therapeutically or if a successful therapy for PAs was found that did not normalize breathing. If an improvement in breathing was invariably linked to a reduction in PAs, to prove that the first caused the second would require demonstrating that the first preceded the second. Of course, if PAs are not accompanied by HV (i.e., our first HV proposition is false), none of these tests is relevant.

There is some evidence that instructions to breathe in a nonhyperventilatory way reduce PAs. Such instructions are an integral part of contemporary psychological treatment packages for PD (Barlow \& Craske, 2000; Wilhelm \& Margraf, 1997), which have been shown to be therapeutically effective. Breathing training has rarely been studied separately from cognitive restructuring, but when it was, it seemed to be effective in reducing panic (Clark, Salkovskis, \& Chalkley, 1985; Salkovskis, Jones, \& Clark, 1986), although severe methodological criticisms of these and other breathing training studies can be raised (Meuret, Wilhelm, Ritz, \& Roth, 2003). Because of technical problems, no one has measured $\mathrm{HV}$ in natural settings long and accurately enough to be able to document in PD patients the occurrence of physiologically defined episodes of HV with panic prior to breathing training or restoration of normal breathing patterns after panic had been eliminated, which would support this proposition and Proposition 2. Our recent results indicate that improving PD patients do indeed learn to raise their resting $\mathrm{pCO}_{2}$ (Meuret, Wilhelm, \& Roth, 2004), but whether this is why they improve is less certain because reattribution of causes of anxiety and changing expectations may have been the active therapy ingredients, and any change in breathing that occurred may have been secondary to anxiety reduction. If a purely nonrespiratory therapy procedure, such as muscle relaxation or cognitive restructuring succeeded in normalizing disturbed breathing, we would suspect that HV is an effect of anxiety rather than a cause.

Pharmacological therapies of PAs could falsify this proposition by eliminating PAs without preventing decrease in $\mathrm{pCO}_{2}$ levels. As far as we know, this has not been tested. The discovery of a substance (including $\mathrm{CO}_{2}$ ) that in proper doses could raise $\mathrm{pCO}_{2}$ levels without suppressing PAs would also be contrary to this proposition.

\section{Heuristic Value}

HV theory has the advantages and disadvantages of providing a single, simple explanation for a multiplicity of clinical and experimental observations. If it were true, it would explain a lot, but Ley (1992a), a major proponent, has seen the need to cede important roles to cognition and learning. $\mathrm{HV}$ theory also struggles with a question unanswered by CCT, namely, Why do individual PAs end. The speculation that motor activity resulting from restlessness or flight restores $\mathrm{pCO}_{2}$ is testable. Why certain drugs alleviate PAs lies completely outside the scope of HV theory, although it has something to say about how the respiratory stimulant doxapram provokes PAs (Abelson et al., 1996).

HV theory seriously challenged other schools of thought about anxiety some decades ago, but now it may be falling out of fashion in spite of a lukewarm embrace by cognitive-behavioral therapists. Many of them are beginning to feel they no longer need it (Schmidt et al., 2000). Whatever its truth, PD patients have probably been helped by its attribution of a mysterious, seemingly physical malady to a bad habit, the voluntary suppression of which 
seems within the reach of the patient. For 50 years, HV theory has stimulated research endeavors, particularly in the last two decades and for some reason, especially in the Netherlands.

\section{SFAT}

\section{Core Thesis}

SFAT was proposed by Klein (1993). Its core thesis is that there is a correspondence between feelings of suffocation or dyspnea and a distinct kind of anxiety occurring in attacks. The feeling of suffocation is a false alarm because the $\mathrm{O} 2$ supply is not actually being compromised. Such attacks are characteristic for PD, whose sufferers experience surges of feelings of breathlessness and are hypersensitive to physical and psychological inducers of those feelings. Deranged physiological regulation of respiration is probably the usual underlying cause of $\mathrm{PD}$, although the specific derangement is as yet unknown.

SFAT does not consider the abnormal sensitivity to rising arterial $\mathrm{pCO}_{2}$ levels, which can be observed in the reaction of $\mathrm{PD}$ patients to inhalation of gasses with higher $\mathrm{CO}_{2}$ concentrations than air, to be the only route to dyspnea and panic. Other asphyxia cues can induce dyspnea and panic. HV from the standpoint of SFAT is a protection against feelings of dyspnea rather than a primary cause of panic. By driving $\mathrm{pCO}_{2}$ far below the threshold for feeling short of breath, the PD patient creates a buffer zone that makes it less likely that the threshold will be exceeded by incidental fluctuations in arterial $\mathrm{pCO}_{2}$.

An important feature of SFAT is that it tries to explain only panic anxiety, which it sharply distinguishes from "anticipatory anxiety." The latter may occur in anticipation of PAs as learned reactions to cues that an attack is imminent. Cues could be entering a shopping mall or airplane, or be bodily sensations that presage an attack. Anticipatory anxiety occurs as a conditioned emotional response to the $\mathrm{PA}$ as an unconditioned stimulus, and can be modeled in animals that predictably receive a shock a few seconds after a cue. In contrast, Klein (1993) speculates that panic anxiety is the specific kind of the anxiety evoked in young animals by separation from their mothers, presenting some evidence that separation anxiety in human children is a precursor to adult PD.

\section{Tests}

Klein's (1993) article cited evidence of many different kinds for his hypothesis (see also Klein, 1994). Some of it, although supportive, is indirect: for example, the relative lack of anxiety in children with a congenital hypoventilation syndrome, an observation that pregnancy protects from panic, reports of "mass hysteria" being precipitated by peculiar odors and the sight of hyperventilating people, and an association of panic with childhood separation. More essential and apparently falsifiable propositions derived from SFAT are the ones below.

1. The occurrence of the feeling of suffocation (dyspnea) can be precisely and objectively established, as can independently, the occurrence of a true PA.

The relationship between dyspnea and $D S M-I V$-defined PAs is circular because 1 of the 13 criteria for panic is dyspnea. In fact, "sensations of shortness of breath or smothering" is one of the most commonly reported criterion symptoms (e.g., Margraf, Taylor, Ehlers, Roth, \& Agras, 1987; McNally, Hornig, \& Donnell, 1995). To avoid circularity, PAs must be redefined by criteria that do not include dyspnea, which would acknowledge the possibility that a true PA in true PD patients might not include dyspnea as a symptom, thus permitting it to be falsified.

Since dyspnea as phrased in the DSM-IV covers more than one subjectively distinguishable kind of difficulty in breathing (Elliott et al., 1991), we might be able to find words that express feelings of suffocation more specifically. For example, "air hunger" may be more pertinent than "shortness of breath" (Simon et al., 1990), but at best we are left dependent on expressions of subjective feelings, which like catastrophic cognitions may or may not be accurately verbalized or verbalizable (Wilhelm \& Roth, 2001). In fact, basing a falsification of SFAT on subjective feelings is probably unfair to it, since its suffocation false alarm is more neurologically primitive than, say, catastrophic cognitions and cannot be restricted to awake, thinking animals. Conscious awareness of the alarm through some kind of interoception is hardly necessary for it to function. Thus, a physiological indicator, like the hypocapnia of HVT, would help enormously in defining the alarm more precisely and objectively, but what indicator should be chosen? Sounding of the alarm cannot be equated with faster or deeper respiration, since these occur automatically to cover normal variations in $\mathrm{O} 2$ requirements depending on energy needs. A drop in $\mathrm{O} 2$ saturation below a certain threshold may indicate impending suffocation, but this kind of hypoxia is unlikely to occur in the daily life of people with normal lung functioning. Moderate hypoxia produces no dyspnea and few acute physiological adjustments and is unlikely to be a concomitant of PAs in panic patients without lung disease. Increases in arterial $\mathrm{pCO}_{2}$ do stimulate increases in ventilation, but these are not invariably accompanied by dyspnea. Thus, not being able to count on blood gasses or lung volume parameters as indicators of dyspnea leaves one with the limitations of self-report discussed above for CCT, which threaten to make propositions derived from SFAT unfalsifiable. The best candidate for a physiological dyspnea measure is perhaps inspiratory flow rate (MilicEmili, Whitelaw, \& Grassino, 1981), which has been observed to be elevated in PD under certain circumstances (Wilhelm, Trabert, \& Roth, 2001b).

2. If a PA occurs naturally, dyspnea will precede or accompany it. If dyspnea occurs naturally, a PA will follow it.

In a study where PD patients kept a panic diary, dyspnea was reported in $37 \%$ of nonsituational attacks having three or more symptoms (Margraf et al., 1987). On the other hand, "palpitations, pounding heart, or accelerated heart rate" were reported in $73 \%$ of these attacks. This heart symptom cluster is ostensibly based on interoception of cardiovascular changes typically accompanying anticipatory anxiety in humans (W. T. Roth, Breivik, Jorgensen, \& Hofmann, 1996) and animals (Fowles, 1980), and so according to SFAT may be irrelevant to true panic anxiety. In any case, the number of panickers not complaining of shortness of breath seems high for SFAT to be true, even if not all panickers report their feelings accurately.

Like catastrophic cognitions and HV, if dyspnea is the cause of PAs, it will be present at the first PA; it will precede or occur concurrently with, rather than follow, individual attacks; and it will 
not occur without being followed by an attack. Like for catastrophic cognitions, falsifying these causal requirements is difficult. An alternative is that dyspnea is an effect rather than a cause (Sinha, Papp, \& Gorman, 2000). According to this scenario, panic patients inherit a sensitive neural fear network that somehow occasionally generates PAs out of the blue. Increased ventilation and dyspnea are innate components of any fear reaction, including panic. Later in the course of the illness, dyspnea itself can trigger panic because it has become a conditioned stimulus to the unconditioned stimulus of the PA (Bouton et al., 2001).

3. Dyspnea is caused naturally by abnormal chemoreceptor mechanisms or indications that $\mathrm{O} 2$ supply might be low.

According to SFAT, a cause of dyspnea in many PAs in PD patients is a tonically or phasically hypersensitive chemoreceptor mechanism. The medullary mechanisms of how arterial gas concentrations and $\mathrm{pH}$ control respiratory parameters have been fairly well worked out in animals (Dempsey \& Pack, 1995), and quantification of this control is feasible in waking and to some extent in sleeping humans. For example, the rate of increase of minute ventilation for a given increase in end-tidal $\mathrm{pCO}_{2}$ can be measured. Most studies examining this rate over a range of $\mathrm{pCO}_{2}$ concentrations have not found it to be greater in PD patients than in controls (for a review, see Sinha et al., 2000). Although these results falsify this proposition for an important mechanism of respiratory regulation, other mechanisms may be abnormal. Panic patients show an irregularity of respiration even at baseline (Wilhelm, Trabert, \& Roth, 2001a; Wilhelm et al., 2001b) and during sleep (Martinez et al., 1996; Stein, Millar, Larsen, \& Kryger, 1995), suggesting some physiological abnormality.

4. Dyspnea is a cause of spontaneous HV in panic patients.

According to SFAT, HV is a reaction to a feeling of dyspnea that functions to prevent that feeling. Falsifying this proposition would require tracking the course of feelings of dyspnea and showing that they occurred before HV. HV is quantifiable in terms of $\mathrm{pCO}_{2}$, but transitory changes in dyspnea, like transitory changes in any emotion or bodily feeling, are difficult to measure sufficiently continuously and accurately to test this proposition. More feasible would be to continuously monitor a physiological cause of dyspnea such as end-tidal $\mathrm{pCO}_{2}$ in panic patients and to determine whether decreases in $\mathrm{pCO}_{2}(\mathrm{HV})$ were regularly preceded by increases in $\mathrm{pCO}_{2}$ beyond that expected by regression to the mean. If this was not the case, and if we accept that increases in $\mathrm{pCO}_{2}$ are a fair proxy for dyspnea, Proposition 4 would be falsified.

5. If dyspnea is induced, a PA will follow. If a true PA is induced, dyspnea will have preceded or accompanied it.

If SFAT is true, dyspnea-inducing procedures such as inhaling gasses with above-air $\mathrm{CO}_{2}$ concentrations or maneuvers like breath holding or breathing through a straw should precipitate PAs (W. T. Roth, Wilhelm, \& Trabert, 1998; Taylor \& Rachman, 1994). Of these, $\mathrm{CO}_{2}$ inhalation is probably most potent, depending on the $\mathrm{CO}_{2}$ concentration and the length of inhalation. For example, in one study, $61 \%$ of PD patients and $8 \%$ of controls rated themselves as having had PAs within $20 \mathrm{~min}$ of beginning to inhale $5 \% \mathrm{CO}_{2}$ (Papp et al., 1997). When lactate infusions do produce PAs, dyspnea is a prominent symptom (Goetz, Klein, \& Gorman, 1996). In asthma or chronic obstructive pulmonary disease, PD has a higher incidence than in other diseases (Goodwin \& Eaton, 2003; Sloan, Shapiro, Bagiella, Gorman, \& Bigger, 1995).

This proposition could be falsified by finding panic patients in whom some procedure has induced dyspnea without inducing panic anxiety or vice versa. Again, it is important to have some independent criterion of true PAs (as distinguished from acute increases in what SFAT calls anticipatory anxiety) so that failure to panic from a dyspnea-inducing procedure is not dismissed as irrelevant to the proposition.

6. If dyspnea is suppressed, a PA will be suppressed. If a PA is suppressed, dyspnea preceding it will have been suppressed.

One way to reduce dyspnea could be to get patients to breathe a little more deeply whenever they start to feel short of breath and whenever they enter an agoraphobic situation where PAs were likely to occur. Of course, this is exactly the opposite of what PD patients are taught in their breathing training, and if that training is successful and if it works by actually changing breathing patterns outside the consultation room (see the discussion of Proposition 4), this proposition would be falsified. However, episodic dyspnea might not be eliminated by a breathing training that tonically increases $\mathrm{pCO}_{2}$, since this could desensitize the suffocation alarm by adaptively raising the set point.

The possibility that pharmacological therapies that are successful in suppressing PAs also suppress the suffocation false alarm and thus feelings of dyspnea is testable. To support SFAT, an antipanic drug should be shown to suppress dyspnea directly by adjusting chemoreceptor mechanisms, leading secondarily to a reduction in PAs, rather than to suppress PAs directly, leading secondarily to a reduction in dyspnea.

\section{Heuristic Value}

The explanatory scope of SFAT is exceedingly broad. The article that launched it knitted together diverse observations from clinical psychiatry, respiratory physiology and pathology, neuroendocrinology, experimental psychology, and behavioral development. SFAT startlingly turns the fundamental premise of HVT on its head. As a "biological" theory, SFAT is opposed in spirit to granting thoughts explanatory status and thus does not care to amplify its conception suffocation fears with elements from CCT. In the last decade many experiments have been conducted to test aspects of this theory, and the introduction and discussion sections of many experimental articles refer to it. In the area of therapy, SFAT's insistence on the distinction of panic anxiety from other kinds of anxiety has had an impact, although it has turned out that successful pharmacological treatments of PD, generalized anxiety disorder, and depression largely involve the same drugs.

\section{$\mathrm{NH}$}

\section{Core Thesis}

In two articles published 11 years apart, Gorman et al. proposed two versions of their NH of PD (Gorman, Kent, Sullivan, \& Coplan, 2000; Gorman, Liebowitz, Fyer, \& Stein, 1989). The first 
tried to localize the three distinct aspects of PD postulated by Klein (1980) - the acute attack, anticipatory anxiety, and phobic avoidance-into three neuroanatomical locations. The seat of the PA was the brainstem loci where serotonergic and noradrenergic pathways regulated respiration and where antipanic drugs such as imipramine have their site of action. Learned or "anticipatory anxiety" was mediated by limbic circuits that could be inhibited by benzodiazepines or relaxation training. Phobic avoidance was mediated by prefrontal activation, which was modifiable by desensitization and cognitive therapies. Neural pathways connecting these three locations explained the clinical relationships observed between these three aspects of PD.

The second, revised model hypothesized that PAs are analogous to a conditioned fear response in animals and are mediated by a fear network centered in the amygdala with connections to the hippocampus, medial prefrontal cortex, and hypothalamus. Thus, the idea that the brainstem is the seat of panic was rejected in favor of larger set of brain locations and circuits. This network is "abnormally sensitive" in PD patients "by virtue of a genetically imposed abnormality." Medications that increase overall transmission among serotonergic nerves normalize it through several mechanisms. Increasing inhibitory input from serotonergic neurons in the brainstem raphe dampens noradrenergic neurons in the locus ceruleus and reduces transmission to the periaqueductal gray area that mediates defense-escape behaviors. Because the brain fear network is more sensitive, traumas such as separation and disrupted attachment lead to manifest PD in susceptible individuals. The hippocampus is the site of contextual learning, which can place fear in specific locations such as shopping malls or together with specific somatic sensations. Psychotherapies affecting panic such as CBT and psychoanalysis, operate upstream from the amygdala at the level of prefrontal cortex and hippocampus.

\section{Tests}

A theory of this breadth should spawn an array of testable propositions, and indeed Gorman et al.'s 1989 article lists nine "experimental tests of the hypothesis," among which are those "required to either validate or disprove our model" (p. 157). They range in methodology from animal experiments and neuroimaging to examinations of psychological treatment outcomes. Some of these seem less critical to its central theses than others. Tests 1 and 2 involve animal experiments. Animal anxiety does have many similarities to human anxiety, but if panic anxiety is one of several kinds of anxiety, it will be necessary to show that anxiety homologous to the characteristic unexpected, apparently uncued anxiety attacks of human PD can be observed in animals. Furthermore, the relevance of animal models to PD depends on how much this disorder depends on complex cognitions. Animal experiments can assess functions that are analogous to some kinds of cognition in humans, such as attention, memory, choice between alternatives, and the evaluation of social situations. Yet, evidence for thoughts in animals of the type expressed by language is meager, even in nonhuman primates. CCT postulates, as a cause of panic, beliefs about possible life-threatening consequences of bodily sensations. Without language, it is hard to see how an animal could entertain such ideas.

Two tests call for purely clinical experiments. Test 6 is whether benzodiazepines block anticipatory anxiety and not panic. Clinical drug studies have not supported this proposition (Ballenger et al., 1988) and thus have falsified the original distinction of Klein (1980) between drugs affecting anticipatory anxiety and panic. Test 8 proposes determining whether agoraphobics successfully treated by CBT will continue to have occasional PAs. The answer is almost certainly yes, since anxiety treatments are never $100 \%$ effective. The authors' argument is that if clinical phenomena of PD are not distinct in having different responses to specific therapies, the brain areas that mediate them will not be distinct. Since the NH accepts and builds on Klein's clinical distinctions, falsifying an element of the latter is regarded as automatically falsifying an element of the former. Tests 4, 5, 7, and 9 postulate that human brain imaging studies will demonstrate increased activation in the hypothesized areas when certain kinds of anxiety are induced and decreased activation when that anxiety is attenuated. Since the unique, central feature of the NH is the correspondence between specific clinical phenomena and specific areas of the brain, such studies are of undeniable relevance for testing this theory.

The principle of falsifiability is reaffirmed in Gorman et al.'s (2000) article: "This model suggests many experimental tests . . many aspects of our revised Neuroanatomical Hypothesis are likely to prove incorrect" (p. 503). An example of a prediction of the revised $\mathrm{NH}$ is that "in at least two ways ... psychotherapy of several types should be useful in treating PD" (p. 502). One is to modify contextual learning in the hippocampus and the other is to reduce sensitivity to "separation, fear of impending doom and death, and overreaction to somatic cues...mediated by higher cortical centers" (p. 502). The authors repeat the promise of imaging for localizing the neurologic substrates for different aspects of panic and for the sites of action of effective therapy. Yet, the revised $\mathrm{NH}$ does not seem to have been based on failures to confirm propositions generated by the original theory. No such failures are mentioned in the revision, and the experimental tests of hypotheses listed in the article are never revisited. Instead, Gorman et al. (1989) were stimulated to revise their theory because the "original version is ... almost completely divorced from exciting preclinical and basic research that has elegantly mapped out the neuroanatomical basis for fear" (p. 494).

We propose the following propositions as central to the revised $\mathrm{NH}$. If they are refutable, they are deductions from valid theorizing, and if they were refuted, they would falsify the NH insofar as it aspires to be a separate theory of panic rather than an amalgam of ideas appropriated from less overarching biological or psychological theories, or a compendium of facts about anxiety.

1. Nonsituational PAs will be accompanied by activation in the amygdala and hypothalamus but not in the hippocampus.

In principle, such a test seems straightforward. Functional magnetic resonance imaging (fMRI) and positron emission tomography (PET) based on glucose utilization are both capable of detecting amygdala and hippocampal activation, although hypothalamic activation is beyond the spatial resolution of current procedures. In the future, however, such resolution may be possible. Managing to capture a nonsituational PA in a scanner is a practical problem, because in many PD patients, these do not even happen every day, but given enough time in the scanner, such attacks might be observed. A theoretical and practical problem is defining a nonsi- 
tuational attack in a way that allows it to be reliably distinguished from a situational attack. Scanners are a claustrophobic environment even for patients without a history of PD (Harris, Robinson, \& Menzies, 1999).

Past attempts to localize complex behaviors to specific brain areas have had both failures and successes, as is apparent from the history of aphasiology (H. L. Roth \& Heilman, 2000). Such localization exemplifies the basic goal of psychophysiology, to achieve a mapping between the psychological and physiological domains. The logic of brain localization of psychological phenomena such as PAs distinguishes several kinds of correspondence. Invariant one-to-one relationships are the exception, and inferences of causality are particularly difficult (Cacioppo, Tassinary, \& Berntson, 2000) but irrelevant to the proposition as stated.

The interpretation of activation in scanner images is not unequivocal. Whether neural firing in a certain area means that that area is exciting anxiety or inhibiting it can be hard to distinguish. Furthermore, neither fMRI nor PET reflects neural firing per se, although the correlation for fMRI may be close (Bandettini \& Ungerleider, 2001). Both may be influenced by the vascular and metabolic effects of HV, as Gorman et al. (2000) pointed out.

2. Situational PAs will be accompanied by hippocampal activation as well.

The feasibility of capturing a situational PA in a scanner is much greater than of capturing a natural, nonsituational one, but both kinds are needed so that they can be compared. Situational attacks are likely in PD patients whose attacks are precipitated by confinement. Lying in the magnet immobilizes the subject and is stressful even for certain people without a history of anxiety disorders (Harris et al., 1999). Physical arrangements could be devised to make the subject's environment even more claustrophobic. The localization of situational PAs is less likely to be disconfirmed than the localization of nonsituational PAs, since hippocampal activation may well occur with both kinds of attacks. Someone having a PA is likely to become sensitive to his or her location in space and what escape routes are open. If perception of spatial context is mediated by the hippocampus, that should be reflected by activation of this structure.

3. Medications suppressing PAs will prevent the activation of the structures activated in natural PAs when PD patients are given provocations.

The NH tries to integrate the sites of action of anxiolytics and the localization of PAs. Proposition 3 is our suggestion of a two-step test of this integration. First it must be shown that provocations such as lactate infusions or $\mathrm{CO}_{2}$ inhalations activate the amygdala and hypothalamus when they induce a PA. Then it must be shown that this activation does not occur if antipanic drugs such as selective serotonin reuptake inhibitors block panic to the provocation. This proposition is based on the assumption that provocations produce true PAs, which could be confirmed by comparing the structures activated by the provocations to those activated naturally by nonsituational PAs. Falsification of the proposition would occur if provocations continued to activate these structures in the absence of PAs. Quite likely panic anxiety is not all-or-none but a matter of degree, so that the relationship between the amount of activation in the relevant structures and amount of anxiety experienced with the provocation needs to be examined before and after treatment with the antipanic medication. One difficulty with falsification is the possibility that medication could suppress structures mediating panic downstream from the source structure. Additional data would be needed to rule this out.

4. Psychotherapies will result in less prefrontal cortical activation when $\mathrm{PD}$ patients are given provocations.

It is essential to distinguish between localizing where psychotherapies act and localizing the specific ideas and beliefs that they change. Insofar as the therapy consists in altering specific ideas such as catastrophic cognitions, imaging will not help because the content of semantic propositions is most probably diffusely distributed in the cortex. We cannot expect to first localize some person's catastrophic thought and then observe its disappearance from an image after psychotherapy has succeeded in eliminating it. Not just thoughts, but other complex psychological processes essential to the construction of fear networks, such as the recording, organizing, storage and retrieval of specific memories, may involve scattered synapses whose localization is beyond the resolution of a scan. In general, the usefulness of neuroanatomical localization for understanding an anxiety disorder diminishes to the extent that the disorder depends on semantic propositions. Learning indeed depends on synaptic changes, but for learning of specific contents, such as French vocabulary, references to brain locations are misdirected. Szasz (1961) made this point convincingly for pseudoneurological conversion symptoms in his book The Myth of Mental Illness.

On the other hand, the general intensity of thinking and planning may be reflected by diffuse frontal lobe activation, in which case Proposition 4 would be a legitimate implication of the NH. PD patients who had had PAs to provocations before psychotherapy but not afterward should show reduction of the frontal activity reflecting catastrophic thinking. Unfortunately for the falsifiability of the $\mathrm{NH}$, this theory might also be construed as implying the opposite, that psychotherapies blocking provocations should result in an increase in frontal activity. If a therapy works by teaching patients to inhibit anxiety by thinking in certain ways, thinking and frontal activity might intensify upon exposure to a provocation. That might be the case if the therapy prescribes active disputing of catastrophic cognitions or active redirection of attention away from the threat. Thus, the specific action of the therapy may have to be taken into account before formulating a testable proposition.

\section{Heuristic Value}

The breadth and inclusiveness of the $\mathrm{NH}$ should encourage a wide range of scientific activity. The $\mathrm{NH}$ considers evidence from diverse research methodologies, and supports interdisciplinary efforts and accepts the contributions of warring theoretical schoolsbiological psychiatry, cognitive-behavioral psychology, and psychoanalysis. The importance of HV is affirmed, and even VCT is afforded a role: selective serotonin reuptake inhibitors, by blocking the physical effects of panic, may act to reduce the anxiety produced by perceiving such effects (Gorman et al., 2000). However, breadth has both virtues and drawbacks. A comprehensive theory must somehow form an integrated whole, explaining diverse phe- 
nomena by a new common principle. Does NH succeed in that, or is it little more than a collection of independent theoretical speculations, whose whole does not succeed in being more than the sum of its parts?

Heuristic value can come from the ability of a theory to generate surprising implications, which if true, would shake the edifice of received truths. This cannot be the case for $\mathrm{NH}$ insofar as it is merely a translation of facts from clinical psychology and pharmacology into neuroanatomy. At best a translation from one language to another does not add or subtract meaning but simply follows the fixed rules dictating the correspondence between them. At worst a translation fails to convey the full meaning of the original. An example of such a translation in NT is that psychosocial treatments affecting panic operate via prefrontal-amygdala connections. That prefrontal cortex is involved must be true because CBT works and because CBT involves words and ideas, which depend on the presence of cortex without more specific functions. That words and ideas affect panic is translated into neuroanatomically established connections between the cortex and deeper lying emotional systems. But what have we gained by this? Because of the high interconnectivity of different parts of the brain, the absence of a plausible pathway would have been more exceptional and thus more informative than the existence of a pathway. Furthermore statements about prefrontal-amygdala connections apply to words and emotions in general, not to PAs specifically, and thus entail a substantial reduction in precision. Until the unlikely event that our neuroanatomy is able to place, for example, thoughts of disaster and thoughts of mastery in distinct centers, translation from psychological concepts of PAs to neuroanatomical pathways will entail a loss in information.

\section{General Conclusions}

The widely cited theories of PAs that we have chosen for evaluation are diverse. The two respiratory theories postulate a unitary biological causal explanation; one, an essentially psychological explanation; and three, explanations unifying biological and psychological explanations. Our judgment of their falsifiability is based on the difficulty of empirical falsification with the experiments that have been designed for that purpose rather than on the original Popperian criterion of formal logical falsifiability. We can imagine decisive falsification tests for all the theories, but for some theories these tests will be so difficult that the theory is likely to be superceded before the tests are carried out. The burden of finding these tests does not fall exclusively on the shoulders of the critic. The formulator of the theory bears ultimate responsibility for its testability.

We have tried to be fair in appraising the falsifiability of both psychological and biological theories. Is that criterion so stringent that it cannot be met by any psychological hypothesis even if it is meaningful and true? After all, the scientific standards of the outcome studies that show that specific CBTs are beneficial are of the highest order, and their therapeutic mechanism is definitely nonbiological, at least in the usual sense of "biological." Biological theories do have an advantage in being simpler if not simplistic, and thus their premises easier to unequivocally falsify, but whenever psychology has sworn off thoughts in favor of strict behaviorism, their reality and power has always reasserted themselves. Some words definitely heal, but which ones and how?
Psychoanalysis, a causal cognitive theory of the last century criticized by Popper as unfalsifiable, asserts that unconscious sexual thoughts cause PAs. Many today reject that claim but accept the claim that catastrophic thoughts cause PAs, probably swayed by the fact that the newer theory is the basis of a therapy with better results than psychoanalysis. But the problem remains that any causal theory depending on specific thoughts is hard to falsify, as our analysis of CCT demonstrated.

What would be the implications for the CCT if noncognitive factors such as electroencephalogram spiking or unperceived internal stimuli related to an infantile emotional learning experience (cf. Jacobs \& Nadel, 1998) were discovered to invariably precede PAs. Or if a gene were found to be highly and specifically associated with having PAs (for a review, see Smoller \& Tsuang, 1998)? These discoveries would not falsify CCT since catastrophic thoughts might still be necessary and sufficient causes for PAs, albeit only a later cause in a chain of causes. Since genes are known to affect temperamental variables like neuroticism and behavioral inhibition, they could set the stage for acute anxiety episodes, enhancing perception of threat and making threatrelevant thoughts more accessible. Thus, PAs could have two necessary causes, a genetic cause and catastrophic thoughts. Both kinds of causes or neither kind might be individually sufficient under normal background conditions. A revised CCT might postulate that catastrophic thoughts serve to perpetuate PAs after the original noncognitive cause was no longer operative, these thoughts having become a sufficient cause through learned associations. Or if the basic cause continued to be operative, a revised theory might assert that catastrophic thoughts are a contributing cause, transforming occurrences of the noncognitive cause from incidental perceptions of mysterious bodily sensations into frightening events with disastrous consequences

Thus, our critical analysis of CCT should not be taken as a blanket rejection of all hypotheses about PD containing thought and belief variables. The problem with the current theory as we read it is the sine qua non causal role for catastrophic thoughts. Probabilistic theoretical formulations of a kind testable by the general linear model, structural equation modeling, and path coefficients are possible and potentially falsifiable. Depending on its statistical relationship to outcome measures, a correlate (factor) can be classified as a mediator, moderator, independent, overlapping, or proxy (Kraemer, 2001). For example, a proposition related to CCT is the following: PA rates will decline as the strength of catastrophic beliefs declines. This is testable because we could devise a questionnaire that would quantify the strength of a patient's beliefs that met an agreed-upon definition of catastrophic, we could ask patients to record the frequency of their attacks, and we could analyze statistically how these two measures changed over time. Falsifiability is replaced by explanatory power. The higher the correlations between measures of the related variables, the more their explanatory power. A low correlation is imaginable: Many patients may continue to believe that the symptoms of panic are dangerous after they have stopped having them; a drug therapy or breathing therapy or meditation might stop attacks without altering catastrophic beliefs much. Our evaluation of this proposition has practical implications: If it is true, monotherapies directly addressing catastrophic beliefs may be the most efficient, while if it is false, competing approaches alone or in combination may be more efficient. Yet the hypothesis is not causal. Cata- 
strophic beliefs could just as easily be an effect of PAs rather than a cause: The strength of those beliefs could decline as a direct result of attacks becoming less frequent.

In spite of the falsifiability problems of CCT, its heuristic value is indisputable. It has confounded the expectations of biologically oriented clinicians by inspiring a psychotherapy whose effectiveness can no longer be doubted, and whose premise that right thinking can overcome irrational human emotion, had long been considered a naive belief of our grandparents. It has turned behavior therapy on its head by making acceptable an adjective with the word "cognitive" standing before "behavioral," nonchalantly connected to it with a dash, giving simple expression to a profound paradigm shift in clinical psychology. The success of cognitive psychotherapies is a compelling reason for not giving up trying to develop a falsifiable psychological theory of pathological anxiety where modification of thoughts makes sense. Of course, their efficacy does not mean that any theory of catastrophic cognitions would be verified; exposure not depending on cognitions may prove to be the essential ingredient in cognitive-behavioral treatments.

VCT is currently discussed principally as a component of the CCT, but it is also applicable to supplement the HVT or the SFAT. Its invariable appearance in therapy manuals for PD attests to a certain heuristic value for clinicians and their patients. On the other hand, as a variation of the old James-Lange theory of emotion, it is hardly new, nor does it provide a comprehensive explanation for PAs, not specifying why the manifestations of anxiety are feared or what properties of the vicious circle finally bring individual attacks to an end. For specific manifestations of anxiety, VCT can be falsified by showing that the induction or suppression of that manifestation fails to suppress PAs, but general falsification of VCT is unfeasible because of the multitude of possible anxiety manifestations and their combinations, any of which could be operative in a given individual.

The TAT is a theory that makes distinctions, and we have concentrated on the validity of these distinctions rather than their causal implications. Falsification by showing that a PA does not belong to a certain category is particularly improbable in the case of false alarms, which is a residual or wastebasket category for anxiety episodes that do not fit elsewhere. In a sense this theory is saying that no one knows what causes the initial attacks that get PD started, an opinion that we share. That later in the course of PD, effects of emotional learning become manifest can scarcely be denied, but whether learned anxiety episodes are identical to nonlearned episodes is less certain. TAT is quite unhelpful when one is faced with the question of whether a specific individual episode should be classified as learned or not. The theory has heuristic value in promoting theoretical learning mechanisms that justify therapeutically effective unlearning and relearning procedures for treating PAs.

HVT is tied to a measurable biological parameter, arterial hypocapnia, which can be estimated even ambulatorily. The equation that the simple form of HVT draws between PAs and episodes of $\mathrm{HV}$ is not only falsifiable, but has been falsified: Hyperventilation often fails to occur when PAs do, and vice versa. Although HV can easily be induced by a few minutes of voluntary overbreathing, this often fails to induce PAs, even in patients susceptible to them. Yet this negative evidence does not mean that HV could not cause panic under certain conditions or be a crucial event in the first attacks in the course of a disorder of repeated attacks. For example, Ley (1992b) speculated that in an initial attack or attacks, acute $\mathrm{HV}$ associated with autonomic activation from stress results in panic when resting $\mathrm{pCO}_{2}$ is already lower than normal because of chronic HV. In principle this elaborated HVT could be falsified by showing that resting $\mathrm{pCO}_{2}$ levels failed to predict first attacks. Other vulnerabilities might also need to be present for HV to cause panic.

Heuristically, HVT has served as a biological alternative and adjunct to psychological explanations of panic for more than half a century, and in the last two decades has stimulated considerable research. It is uncertain whether therapies that try to teach people not to hyperventilate actually succeed in doing that, but this does not preclude the possibility of their being beneficial. Recent studies have confirmed that PD patients do indeed exhibit respiratory peculiarities, albeit not exactly the ones predicted by HVT (Wilhelm, Gerlach, \& Roth, 2001; Wilhelm et al., 2001a, 2001b).

The SFAT, the other respiratory theory of panic, is difficult to falsify because of its failure to identify an abnormal physiological respiration mechanism underlying the feelings of dyspnea in PD patients during PAs. Having to rely on self-report gives this biologically oriented theory all the problems of the CCT, which is ready and able to include fear of feelings of suffocation as another possible catastrophic cognition. A second major barrier to falsification is the restriction of the theory to true PAs in true PD patients. To prevent this being a loophole preventing disconfirmation, definitions of PAs and PD totally independent of the presence of respiratory distress are needed. Reliably distinguishing panic anxiety from anticipatory anxiety may be impossible because the symptoms and physiological characteristics of these supposedly different kinds of anxiety are highly similar if not identical. In any case, the SFAT has been an undeniable success heuristically, turning upside down the established HVT, challenging psychological explanations of PAs and PD, and inspiring numerous psychobiological investigations.

NH differs from the other theories in its breadth, attempting to integrate data from neuroanatomy, neurophysiology, pharmacology, and psychology. These data were collected originally in order to test narrower but also comprehensive theories, including versions of those considered here, particularly SFAT. Its structure makes it unsusceptible to falsification as a whole; at most, a component might be falsified, stimulating an addendum or revision. We have restricted ourselves here to the falsifiability of propositions that represent its most unique feature, hypothesized correspondences between clinical phenomena and brain areas. The correspondences are falsifiable by brain imaging in principle, although specific testing problems mentioned in the 2000 revision-limitations in our ability to visualize hypothalamic nuclei; to capture transient, unpredictable PAs in the scanner; and to discount nonspecific effects of $\mathrm{HV}$ on brain blood flow-are barriers to falsifiability in practice. The specific testing problems mentioned in the 2000 revision could well be surmountable in the not too distant future. More fundamental than these technical problems, however, is the possibility that brain localization may not advance our understanding of panic very far because of the activation of multiple brain regions each with multiple functions. Succeeding in locating in brain space by any kind of imaging, specific semantic structures that cause panic is highly unlikely. 
$\mathrm{NH}$ has the positive heuristic attribute of comprehensiveness but lacks the elements of unexpectedness and challenge, because it consists largely of accepted opinions translated into neurologic language. A translation ordinarily entails some loss in information, although reformulating old findings in a way that transcends the boundaries of a single scientific discipline might stimulate the collection of new data.

Table 1 summarizes our conclusions about the falsifiability and heuristic value of the six theories considered. Falsifiability here must be understood in the non-Popperian sense of whether current research approaches for evaluating the theories are potentially capable of falsifying them, not whether the theories are falsifiable in principle. In the absence of a recognized, objective measure of heuristic value, we have relied on our subjective impressions of how often we have heard the theory discussed at scientific meetings, how often it came up in graduate seminars, and how it is treated in textbooks and reviews. One quantitative measure is possible for four of the theories, namely the number of times the key article articulating the theory has been cited, which is listed in Table 2. These counts are based on a search of the SciSearch Plus databases. We admit that this measure of heuristic value is far from perfect. For example, repeated citation may merely signify appeal to the authority of a received opinion. On the other hand, outrageous, contrarian articles can attract attention without setting any productive new direction, although if they have passed peer review, they may usefully cause proponents of a theory to reexamine their premises. Citations can be high for articles that are more innovative methodologically than theoretically, although better measurements may serve to disprove conventional wisdom. Investigators with more funding and more students can generate more citations than investigators with little funding, and granting committees tend to be reluctant to give money for research that deviates very far from the beaten track. Furthermore, the number of citations will vary depending on how long an article has been published, and whether it is well represented by single articles. For this last reason we have not included the vicious circle or the TAT in our tabulation. In addition, our choice of a single article for HVT probably underestimates its influence. However, for the other theories the count of citations is relevant to how much excitement the theories have generated.

Finally, it is important to reiterate that the expansion of scientific knowledge that we witness at an ever accelerating pace is hardly ever the result of systematic testing of individual theories that are definitively discarded when a test falsifies one of their elements. The theories we have considered are too interesting to be

Table 1

Evaluation Summary

\begin{tabular}{lll}
\hline \multicolumn{1}{c}{ Theory } & \multicolumn{1}{c}{ Falsifiable? } & Heuristic Value \\
\hline Catastrophic cognitions & $\begin{array}{c}\text { Unlikely if specific ideas } \\
\text { are considered causal }\end{array}$ & Very much \\
Vicious circle & Not in its present form & Somewhat \\
Three alarms & Not in its present form & $\begin{array}{l}\text { Somewhat } \\
\text { Hyperventilation }\end{array}$ \\
$\begin{array}{l}\text { Suffocation false alarm } \\
\text { Neuroanatomical }\end{array}$ & $\begin{array}{l}\text { Only if better specified } \\
\text { Possibly if specific ideas } \\
\text { are not essential }\end{array}$ & $\begin{array}{l}\text { Vory much } \\
\text { Somewhat }\end{array}$ \\
\hline
\end{tabular}

Table 2

Number of Citations

\begin{tabular}{llc}
\hline \multicolumn{1}{c}{ Theory } & \multicolumn{1}{c}{ Article } & No. of citations \\
\hline Catastrophic cognitions & Clark (1986) & 670 \\
Hyperventilation & Ley (1985a) & 99 \\
Suffocation false alarm & Klein (1993) & 320 \\
Neuroanatomical & Gorman et al. (1989) & 240 \\
\hline
\end{tabular}

Note. Search was conducted via SciSearch Plus, including Social SciSearch (1973-2004), and Arts \& Humanities (1975-2004), as of December 31, 2003.

consigned to the garbage heap on the basis of a few disconfirming experiments. Proponents of the theories will and should try to rescue them with modifications and addenda. A demonstration that catastrophic thoughts are not necessary and sufficient causes of PAs does not imply that such thoughts do not play a major role in the maintenance of PAs. HVT may not be generally true, but its value becomes apparent when a clinician is confronted in the emergency room of a hospital with a panicked patient breathing rapidly with tingling fingers. Since the data inspiring these theories must somehow be explained, we expect the theories considered here to continue in revised forms, perhaps as elements of theories yet to be devised.

\section{References}

Abelson, J., \& Nesse, R. (1994). Pentagastrin infusions in patients with panic disorder: I. Symptoms and cardiovascular responses. Biological Psychiatry, 36, 465-466.

Abelson, J. L., Nesse, R. M., Weg, J. G., \& Curtis, G. C. (1996). Respiratory psychophysiology and anxiety: Cognitive intervention in the doxapram model of panic. Psychosomatic Medicine, 58, 302-313.

Alpers, G. W., Wilhelm, F. H., \& Roth, W. T. (2001). Does one circle fit all? Evidence for the vicious circle model for panic attacks. Manuscript in preparation.

American Psychiatric Association. (1994). Diagnostic and statistical manual of mental disorders (4th ed.). Washington, DC: Author.

Aronson, T. A., Whitaker-Azmitia, P., \& Caraseti, I. (1989). Differential reactivity to lactate infusions: The relative role of biological, psychological, and conditioning variables. Biological Psychiatry, 25(4), 469481.

Ballenger, J. C., Burrows, G. D., DuPont, R. L., Lesser, I. M., Noyes, R. J., Pecknold, J. C., et al. (1988). Alprazolam in panic disorder and agoraphobia: Results from a multicenter trial: I. Efficacy in short-term treatment. Archives of General Psychiatry, 45, 413-422.

Bandettini, P., \& Ungerleider, L. (2001). From neuron to BOLD: New connections. Nature Neuroscience, 4, 864-866.

Barlow, D. H. (1988). Anxiety and its disorders: The nature and treatment of anxiety and panic. New York: Guilford Press.

Barlow, D. H. (1997). Cognitive-behavioral therapy for panic disorder: Current status. Journal of Clinical Psychiatry, 58(Suppl. 2), 32-37.

Barlow, D. H. (2002). Anxiety and its disorders: The nature and treatment of anxiety and panic (2nd ed.). New York: Guilford Press.

Barlow, D., \& Craske, M. (2000). Mastery of your anxiety and panic (3rd ed.). Boulder, CO: Graywind.

Baum, M. (1986). An animal model for situational panic attacks. Behaviour Research and Therapy, 24, 509-512.

Bouton, M. E., Mineka, S., \& Barlow, D. H. (2001). A modern learning theory perspective on the etiology of panic disorder. Psychological Review, 108, 4-32. 
Brewin, C. (2001). A cognitive neuroscience account of posttraumatic stress disorder and its treatment. Behaviour Research and Therapy, 39, 373-393.

Brown, T. A., Chorpita, B. F., \& Barlow, D. H. (1998). Structural relationships among dimensions of the $D S M-I V$ anxiety and mood disorders and dimensions of negative affect, positive affect, and autonomic arousal. Journal of Abnormal Psychology, 107, 179-192.

Cacioppo, J. T., Tassinary, L. G., \& Berntson, G. G. (2000). Psychophysiological science: Handbook of psychophysiology (2nd ed., pp. 3-23). Cambridge, England: Cambridge University press.

Clark, D. M. (1986). A cognitive approach to panic. Behaviour Research and Therapy, 24, 461-470.

Clark, D. M. (1996). Panic disorder: From theory to therapy. In P. M. Salkovskis (Ed.), Frontiers of cognitive therapy (pp. 318-344). New York: Guilford Press.

Clark, D. M., \& Ehlers, A. (1993). An overview of the cognitive theory and treatment of panic disorder. Applied and Preventative Psychology, 2, 131-139.

Clark, D. M., \& Hemsley, D. R. (1982). The effects of hyperventilation: Individual variability and its relation to personality. Journal of Behavior Therapy and Experimental Psychiatry, 13, 41-47.

Clark, D. M., Salkovskis, P. M., \& Chalkley, A. J. (1985). Respiratory control as a treatment for panic attacks. Journal of Behavior Therapy and Experimental Psychiatry, 16, 23-30.

Clark, D. M., Salkovskis, P. M., Hackmann, A., Middleton, H., Anastasiades, P., \& Gelder, M. (1994). A comparison of cognitive therapy, applied relaxation and imipramine in the treatment of panic disorder. British Journal of Psychiatry, 164, 759-769.

Clark, D. M., Salkovskis, P. M., Öst, L.-G., Breitholtz, E., Koehler, K. A., Westling, B. E., et al. (1997). Misinterpretation of body sensations in panic disorder. Journal of Consulting and Clinical Psychology, 65, 203-213.

Cox, B. J., Borger, S. C., \& Enns, M. W. (1999). Anxiety sensitivity and emotional disorders: Psychometric studies and their theoretical implications. In S. Taylor (Ed.), Anxiety sensitivity: Theory, research, and treatment of the fear of anxiety (pp. 115-148). Mahwah, NJ: Erlbaum.

Craske, M. G., \& Freed, S. (1995). Expectations about arousal and nocturnal panic. Journal of Abnormal Psychology, 104, 567-575.

Craske, M. G., Lang, A. J., Rowe, M., DeCola, J. P., Simmons, J., Mann, C., et al. (2002). Presleep attributions about arousal during sleep: Nocturnal panic. Journal of Abnormal Psychology, 111, 53-62.

Dempsey, J. A., \& Pack, A. I. (1995). Regulation of breathing. New York: Marcel Dekker.

Ehlers, A., \& Breuer, P. (1996). How good are patients with panic disorder at perceiving their heartbeats? Biological Psychology, 42, 165-182.

Ehlers, A., \& Clark, D. (2000). A cognitive model of posttraumatic stress disorder. Behaviour Research and Therapy, 38, 319-345.

Ehlers, A., Margraf, J., Roth, W. T., Taylor, C. B., \& Birbaumer, N. (1988). Anxiety induced by false heart rate feedback in patients with panic disorder. Behaviour Research and Therapy, 26, 1-11.

Elliott, M. W., Adams, L., Cockcroft, A., MacRae, K. D., Murphy, K., \& Guz, A. (1991). The language of breathlessness: Use of verbal descriptors by patients with cardiopulmonary disease. American Review of Respiratory Disease, 144, 826-832.

Fowles, D. C. (1980). The three arousal model: Implications of Gray's two-factor learning theory for heart rate, electrodermal activity, and psychopathy. Psychophysiology, 17, 87-104.

Garfield, E. (1992). A citationist perspective of psychology: I. Most cited papers, 1986-1990. American Psychological Society Observer, 5(6), $8-9$.

Garssen, B., Buikhuisen, M., \& van Dyck, R. (1996). Hyperventilation and panic attacks. American Journal of Psychiatry, 153, 513-518.

Goetz, R., Klein, D., \& Gorman, J. (1996). Symptoms essential to the experience of sodium lactate-induced panic. Neuropsychopharmacology, 14, 355-366.

Goldstein, A., \& Chambless, D. (1978). A reanalysis of agoraphobia. Behavior Therapy, 9, 47-59.

Goodwin, R. D., \& Eaton, W. W. (2003). Asthma and the risk of panic attacks among adults in the community. Psychological Medicine, 33 , $879-885$

Gorman, J., Battista, D., Goetz, R., Dillon, D., Liebowitz, M., Fyer, A., et al. (1989). A comparison of sodium bicarbonate and sodium lacatate infusion in the induction of panic attacks. Archives of General Psychiatry, 46, 145-150.

Gorman, J. M., Fyer, M. R., Goetz, R., Askanazi, J., Liebowitz, M. R., \& Fyer, A. J. (1988). Ventilatory physiology of patients with panic disorder. Archives of General Psychiatry, 45, 31-39.

Gorman, J. M., Kent, J. M., Sullivan, G. M., \& Coplan, J. D. (2000) Neuroanatomical hypothesis of panic disorder, revised. American Journal of Psychiatry, 157, 493-505.

Gorman, J. M., Liebowitz, M. R., Fyer, A. J., \& Stein, J. (1989). A neuroanatomical hypothesis for panic disorder. American Journal of Psychiatry, 146, 148-151.

Hardonk, H. J., \& Beumer, H. M. (1979). Hyperventilation syndrome. In P. J. Vinken \& G. W. Bruyn (Eds.), Handbook of clinical neurology (Vol. 38, pp. 309-360). Amsterdam: North-Holland.

Harris, L. M., Robinson, J., \& Menzies, R. G. (1999). Evidence for fear of restriction and fear of suffocation as components of claustrophobia. Behaviour Research and Therapy, 37, 155-159.

Hibbert, G., \& Pilsbury, D. (1989). Hyperventilation-Is it a cause of panic attacks? British Journal of Psychiatry, 155, 805-809.

Hofmann, S. G. (2003). The cognitive model of panic revisited. In M. A. Reinecke \& D. A. Clark (Eds.), Cognitive therapy across the lifespan (pp. 117-137). Cambridge, England: Cambridge University Press.

Hornsveld, H. K., Garssen, B., Fiedeldij Dop, M. J. C., van Spiegel, P., \& de Haes, J. C. J. M. (1996, July 20). Double-blind placebo-controlled study of the hyperventilation provocation test and the validity of the hyperventilation syndrome. Lancet, 348, 152-158.

Jacobs, W. J., \& Nadel, L. (1998). Neurobiology of reconstructed memory. Psychology of Public Policy and Law, 4, 1110-1134.

Jacobs, W. J., \& Nadel, L. (1999). The first panic attack: A neurobiological theory. Canadian Journal of Experimental Psychology, 53, 92-107.

Joiner, T. E., Jr., Steer, R. A., Beck, A. T., Schmidt, N. B., Rudd, M. D., \& Catanzaro, S. J. (1999). Physiological hyperarousal: Construct validity of a central aspect of the tripartite model of depression and anxiety. Journal of Abnormal Psychology, 108, 290-298.

Kenardy, J., Fried, L., Kraemer, H. C., \& Taylor, C. B. (1992). Psychological precursors of panic attacks. British Journal of Psychiatry, 160, $668-673$

Kenardy, J., \& Taylor, C. B. (1999). Expected versus unexpected panic attacks: A naturalistic prospective study. Journal of Anxiety Disorders, 13, 435-445.

Klein, D. F. (1980). Anxiety reconceptualized. Comprehensive Psychiatry, 21, 411-427.

Klein, D. F. (1993). False suffocation alarms, spontaneous panics, and related conditions. An integrative hypothesis. Archives of General Psychiatry, 50, 306-317.

Klein, D. F. (1994). Testing the suffocation false alarm theory of panic disorder. Anxiety, 1, 1-7.

Klein, D. F., \& Klein, H. M. (1989). The definition and psychopharmacology of spontaneous panic and phobia. In P. Tyrer (Ed.), Psychopharmacology of anxiety (pp. 135-162). New York: Oxford University Press.

Kraemer, H. (2001). How do risk factors work together? Mediators, moderators, and independent, overlapping, and proxy risk factors. American Journal of Psychiatry, 158, 848-856.

Kuhn, T. S. (1977). Objectivity, value judgment, and theory choice. In T. S. 
Kuhn (Ed.), The essential tension: Selected studies in scientific tradition and change (pp. 320-339). Chicago: University of Chicago Press.

Lang, P. (1988). What are the data of emotion? In V. Hamilton, G. H. Bower, \& N. Frijda (Eds.), Cognitive perspectives on emotion and motivation (pp. 173-191). Boston: Martinus Nijhoff.

Ley, R. (1985a). Agoraphobia, the panic attack, and the hyperventilation syndrome. Behaviour Research and Therapy, 23, 79-81.

Ley, R. (1985b). Blood, breath, and fears: A hyperventilation theory of panic attacks and agoraphobia. Clinical Psychology Review, 5, 271-285.

Ley, R. (1987). Panic disorder: A hyperventilation interpretation. In L. Michaelson \& M. Ascher (Eds.), Cognitive-behavioral assessment and treatment of anxiety disorders (pp. 191-212). New York: Guilford Press.

Ley, R. (1989). Dyspneic-fear and catastrophic cognitions in hyperventilatory panic attacks. Behaviour Research and Therapy, 27, 549-554.

Ley, R. (1992a). A hyperventilation interpretation of the termination of panic attacks: A reply to van den Hout, de Jong, Zandbergen and Merckelbach. Behaviour Research and Therapy, 30, 191-192.

Ley, R. (1992b). The many faces of Pan: Psychological and physiological differences among three types of panic attacks. Behaviour Research and Therapy, 30, 347-357.

Mackie, J. L. (1965). Causes and conditions. American Philosophical Quarterly, 2, 245-264.

Maddock, R. J., \& Carter, C. S. (1991). Hyperventilation-induced panic attacks in panic disorder with agoraphobia. Biological Psychiatry, 29, 843-854.

Margraf, J., Ehlers, A., Herber, B., Meisner, K., \& Wrobel, F. (1991). Hyperventilation, Angstanfälle und soziale Ängste [Hyperventilation, anxiety attacks, and social anxieties]. Verhaltenstherapie, 1, 34-46.

Margraf, J., Ehlers, A., \& Roth, W. T. (1986). Biological models of panic disorder and agoraphobia: A review. Behaviour Research and Therapy, $24,553-567$.

Margraf, J., Taylor, C. B., Ehlers, A., Roth, W. T., \& Agras, W. S. (1987). Panic attacks in the natural environment. Journal of Nervous and Mental Disease, 175, 558-565.

Martinez, J., Papp, L., Coplan, J., Anderson, D., Mueller, C., Klein, D., \& Gorman, J. (1996). Ambulatory monitoring of respiration in anxiety. Anxiety, 2, 296-302.

McNally, R. J. (1994). Panic disorder: A critical analysis. New York: Guilford Press.

McNally, R. J. (1999). Theoretical approaches to the fear of anxiety. In S. Taylor (Ed.), Anxiety sensitivity: Theory, research, and treatment of the fear of anxiety (pp. 3-16). Mahwah, NJ: Erlbaum.

McNally, R. J. (2001). On the scientific status of cognitive appraisal models of anxiety disorder. Behaviour Research and Therapy, 39, 513521.

McNally, R. J., Hornig, C. D., \& Donnell, C. D. (1995). Clinical vs. nonclinical panic: A test of the suffocation false alarm theory. Behaviour Research and Therapy, 33, 127-131.

Meuret, A. E., Wilhelm, F. H., Ritz, T., \& Roth, W. T. (2003). Breathing training for treating panic disorder. Useful intervention or impediment? Behavior Modification, 27, 731-754.

Meuret, A. E., Wilhelm, F. H., \& Roth, W. T. (2004). Effects of capnometry-assisted breathing therapy on symptoms and respiration in panic disorder. Manuscript submitted for publication.

Milic-Emili, J., Whitelaw, W. A., \& Grassino, A. E. (1981). Measurement and testing of respiratory drive. In T. F. Hornbein (Ed.), Regulation of breathing: II (pp. 675-743). New York: Marcel Dekker.

Miller, J., Fletcher, K., \& Kabat-Zinn, J. (1995). Three-year follow-up and clinical implications of a mindfulness meditation-based stress reduction intervention in the treatment of anxiety disorders. General Hospital Psychiatry, 17, 192-200.

Munjack, D., Crocker, B., Cabe, D., Brown, R., Usigli, R., Zulueta, A., et al. (1989). Alprazolam, propranolol, and placebo in the treatment of panic disorder and agoraphobia with panic attacks. Journal of Clinical Psychopharmacology, 9, 22-27.

Nutt, D. J., Glue, P., Lawson, C., \& Wilson, S. (1990). Flumazenil provocation of panic attacks: Evidence for altered benzodiazepine receptor sensitivity in panic disorder. Archives of General Psychiatry, 47, 917-925.

Otto, M., \& Reilly-Harrington, N. (1999). Theoretical approaches to the fear of anxiety. In S. Taylor (Ed.), Anxiety sensitivity: Theory, research, and treatment of the fear of anxiety (pp. 3-16). Mahwah, NJ: Erlbaum.

Papp, L. A., Martinez, J. M., Klein, D. F., Coplan, J. D., Norman, R. G., Cole, R., et al. (1997). Respiratory psychophysiology of panic disorder: Three respiratory challenges in 98 subjects. American Journal of Psychiatry, 154, 1557-1565.

Popper, K. R. (1959). The logic of scientific discovery. New York City: Basic Books.

Poulton, R., \& Menzies, R. G. (2002). Non-associative fear acquisition: A review of the evidence from retrospective and longitudinal research. Behaviour Research and Therapy, 40, 127-149.

Putnam, H. (1974). The "corroboration" of theories. In P. A. Schilpp (Ed.), The philosophy of Karl Popper (Vol. 14, pp. 221-240). LaSalle, IL: Open Court.

Quine, W. V. (1951). Two dogmas of empiricism. Philosophical Review, 60, 20-43.

Rachman, S., Lopatka, C., \& Levitt, K. (1988). Experimental analyses of panic: II. Panic patients. Behaviour Research and Therapy, 26, 33-40.

Razran, G. (1961). The observable unconscious and the inferable conscious in current Soviet psychophysiology: Interoceptive condition, semantic conditioning, and the orienting reflex. Psychological Review, 68, 81150.

Ross, T. (1937). The common neuroses: Their treatment by psychotherapy. An introduction to psychological treatment for students and practitioners (2nd ed.). Baltimore: Wood.

Roth, H. L., \& Heilman, K. M. (2000). Aphasia: A historical perspective. In S. E. Nadeau, L. J. Gonzalez-Rothi, \& B. A. Crosson (Eds.), Aphasia and language: Theory to practice (pp. 3-28). New York: Guilford Press.

Roth, W. T., Breivik, G., Jorgensen, P. E., \& Hofmann, S. (1996). Activation in novice and expert parachutists while jumping. Psychophysiology, 33, 63-72.

Roth, W. T., Wilhelm, F. H., \& Trabert, W. (1998). Voluntary breath holding in panic and generalized anxiety disorders. Psychosomatic Medicine, 60, 671-679.

Roth, W. T., \& Yalom, I. D. (Eds.). (1997). Treating anxiety disorders. San Francisco: Jossey-Bass.

Salkovskis, P. M., Jones, D. R., \& Clark, D. M. (1986). Respiratory control in the treatment of panic attacks: Replication and extension with concurrent measurement of behaviour and $\mathrm{pCO}_{2}$. British Journal of Psychiatry, 148, 526-532.

Schmidt, N. B. (1999). Prospective evaluations of anxiety sensitivity. In S. Taylor (Ed.), Anxiety sensitivity: Theory, research, and treatment of the fear of anxiety (pp. 217-235). Mahwah, NJ: Erlbaum.

Schmidt, N. B., Trakowski, J. H., \& Staab, J. P. (1997). Extinction of panicogenic effects of a $35 \% \mathrm{CO}_{2}$ challenge in patients with panic disorder. Journal of Abnormal Psychology, 106, 630-638.

Schmidt, N. B., Woolaway-Bickel, K., Trakowski, J., Santiago, H., Storey, J., Koselka, M., \& Cook, J. (2000). Dismantling cognitive-behavioral treatment for panic disorder: Questioning the utility of breathing retraining. Journal of Consulting and Clinical Psychology, 68, 417-424.

Simon, P. M., Schwartzstein, R. M., Weiss, J. W., Fencl, V., Teghtsoonian, M., \& Weinberger, S. E. (1990). Distinguishable types of dyspnea in patients with shortness of breath. American Review of Respiratory Disease, 142, 1009-1014.

Sinha, S. S., Coplan, J. D., Pine, D. S., Martinez, J. A., Klein, D. F., \& Gorman, J. M. (1999). Panic induced by carbon dioxide inhalation and 
lack of hypothalamic-pituitary-adrenal axis activation. Psychiatry Research, 86, 93-98.

Sinha, S., Papp, L. A., \& Gorman, J. M. (2000). How study of respiratory physiology aided our understanding of abnormal brain function in panic disorder. Journal of Affective Disorders, 61, 191-200.

Sloan, R., Shapiro, P., Bagiella, E., Gorman, J., \& Bigger, J. J. (1995). Temporal stability of heart period variability during a resting baseline and in response to psychological challenge. Psychophysiology, 32, 191196.

Smoller, J., \& Tsuang, M. (1998). Panic and phobic anxiety: Defining phenotypes for genetic studies. American Journal of Psychiatry, 155, $1152-1162$

Stein, M. B., Millar, T. W., Larsen, D. K., \& Kryger, M. H. (1995). Irregular breathing patterns during sleep in patients with panic disorder. American Journal of Psychiatry, 152, 1168-1173.

Stein, M. B., \& Rapee, R. M. (1999). Biological aspects of anxiety sensitivity: Is it all in the head? In S. Taylor (Ed.), Anxiety sensitivity: Theory, research, and treatment of the fear of anxiety (pp. 199-215). Mahwah, NJ: Erlbaum.

Ströhle, A., Kellner, M., Holsboer, F., \& Wiedemann, K. (1999). Behavioral, neuroendocrine, and cardiovascular response to flumazenil: No evidence for an altered benzodiazepine receptor sensitivity in panic disorder. Biological Psychiatry, 45, 321-326.

Szasz, T. S. (1961). The myth of mental illness. New York: Dell.

Taylor, S. (Ed.). (1999). Anxiety sensitivity: Theory, research, and treatment of the fear of anxiety. Mahwah, NJ: Erlbaum.

Taylor, S., \& Rachman, S. (1994). Klein's suffocation theory of panic [letter; comment]. Archives of General Psychiatry, 51, 505-506.

Uhde, T. W. (2000). Anxiety disorders. In T. R. W. D. M. Kryger (Ed.), Principles and practice of sleep medicine (3rd ed., pp. 1123-1139). Philadelphia: W. B. Saunders.

Vaitl, D. (1996). Interoception. Biological Psychology, 42, 1-27. van Zijderveld, G., Veltman, D., van Dyck, R., \& van Doornen, L. (1999). Epinephrine-induced panic attacks and hyperventilation. Journal of Psychiatry Research, 33, 73-78.

Wilhelm, F. H., Alpers, G. W., Meuret, A. E., \& Roth, W. T. (2001). Respiratory pathophysiology of clinical anxiety outside the laboratory: Assessment of end-tidal $\mathrm{pCO}_{2}$, respiratory pattern variability, and transfer function RSA. In J. Fahrenberg (Ed.), Progress in ambulatory assessment (pp. 313-343). Göttingen, Germany: Hogrefe \& Huber.

Wilhelm, F. H., Gerlach, A. L., \& Roth, W. T. (2001). Slow recovery from voluntary hyperventilation in panic disorder. Psychosomatic Medicine, 63, 638-649.

Wilhelm, F. H., \& Margraf, J. (1997). A cognitive-behavioral treatment package for panic disorder with agoraphobia. In W. T. Roth (Ed.), Treating anxiety (pp. 205-244). San Francisco: Jossey-Bass.

Wilhelm, F. H., \& Roth, W. T. (2001). The somatic symptom paradox in $D S M-I V$ anxiety disorders: Suggestions for a clinical focus in psychophysiology. Biological Psychology, 57, 1-3.

Wilhelm, F. H., Trabert, W., \& Roth, W. T. (2001a). Characteristics of sighing in panic disorder. Biological Psychiatry, 49, 606-614.

Wilhelm, F. H., Trabert, W., \& Roth, W. T. (2001b). Physiological instability in panic disorder and generalized anxiety disorder. Biological Psychiatry, 49, 596-605.

Wolpe, J., \& Rowan, V. C. (1988). Panic disorder: A product of classical conditioning. Behaviour Research and Therapy, 26, 441-450.

Zucker, D., Taylor, D. B., Brouillard, M., Ehlers, A., Margraf, J., Telch, M., et al. (1989). Cognitive aspects of panic attacks: Content, course, and relationship to laboratory stressors. British Journal of Psychiatry, $155,86-91$

Received February 26, 2002

Revision received April 15, 2004

Accepted April 20, 2004 